\title{
CENTRAL DISCONTINUOUS GALERKIN METHODS ON OVERLAPPING CELLS WITH A NON-OSCILLATORY HIERARCHICAL RECONSTRUCTION
}

\author{
YINGJIE LIU, CHI-WANG SHU, EITAN TADMOR, AND MENGPING ZHANG
}

\begin{abstract}
The central scheme of Nessyahu and Tadmor [J. Comput. Phys, 87 (1990)] solves hyperbolic conservation laws on a staggered mesh and avoids solving Riemann problems across cell boundaries. To overcome the difficulty of excessive numerical dissipation for small time steps, the recent work of Kurganov and Tadmor [J. Comput. Phys, 160 (2000)] employs a variable control volume, which in turn yields a semi-discrete non-staggered central scheme. Another approach, which we advocate here, is to view the staggered meshes as a collection of overlapping cells and to realize the computed solution by its overlapping cell averages. This leads to a simple technique to avoid the excessive numerical dissipation for small time steps [Y. Liu; J. Comput. Phys, 209 (2005)]. At the heart of the proposed approach is the evolution of two pieces of information per cell, instead of one cell average which characterizes all central and upwind Godunov-type finite volume schemes. Overlapping cells lend themselves to the development of a central-type discontinuous Galerkin (DG) method, following the series of work by Cockburn and Shu [J. Comput. Phys. 141 (1998)] and the references therein.

In this paper we develop a central DG technique for hyperbolic conservation laws, where we take advantage of the redundant representation of the solution on overlapping cells. The use of redundant overlapping cells opens new possibilities, beyond those of Godunov-type schemes. In particular, the central DG is coupled with a novel reconstruction procedure which removes spurious oscillations in the presence of shocks. This reconstruction is motivated by the moments limiter of Biswas, Devine and Flaherty [Appl. Numer. Math. 14 (1994)], but is otherwise different in its hierarchical approach. The new hierarchical reconstruction involves a MUSCL or a second order ENO reconstruction in each stage of a multi-layer reconstruction process without characteristic decomposition. It is compact, easy to implement over arbitrary meshes and retains the overall pre-processed order of accuracy while effectively removes spurious oscillations around shocks.
\end{abstract}

\section{CONTEnTS}

1. Introduction

2. Central Schemes on Overlapping Cells

3. A Central Discontinuous Galerkin Method on Overlapping Cells for Conservation Laws

3.1. Numerical Errors for Smooth Solutions

4. A General Non-Oscillatory Hierarchical Reconstruction Procedure

4.1. An Example for Piece-wise Quadratic Finite Element Space in 1D

4.2. Hierarchical Reconstruction - General Description

4.3. Implementation for Piece-wise Quadratic Finite Element Space in 2D 16

5. Additional Numerical Examples 18

6. Concluding Remarks and a Plan for Future Work 22

References

Date: April 16, 2007.

Key words and phrases. Central scheme, discontinuous Galerkin method, ENO scheme, MUSCL scheme, TVD scheme.

Acknowledgment. The research of Y. Liu was supported in part by NSF grant DMS-0511815. The research of C.-W. Shu was supported in part by the Chinese Academy of Sciences while this author was visiting the University of Science and Technology of China (grant 2004-1-8) and the Institute of Computational Mathematics and Scientific/Engineering Computing. Additional support was provided by ARO grant W911NF-04-1-0291 and NSF grant DMS-0510345. The research of E. Tadmor was supported in part by NSF grant 04-07704 and ONR grant N00014-91-J-1076. The research of M. Zhang was supported in part by the Chinese Academy of Sciences grant 2004-1-8. 


\section{INTRODUCTION}

The first-order Godunov and Lax-Friedrichs (LxF) schemes are, respectively, the forerunners for the large classes of upwind and central high-resolution schemes for nonlinear conservation laws and related equations. The Godunov scheme captures shock waves monotonically in narrow transition layers. It is based on evolving a piece-wise cell average representation of the solution, by evaluating the fluxes at the boundaries of each cell which are obtained from the solution of (approximate) Riemann problems along the boundary interfaces. Various higher-order generalizations of Godunov scheme have been developed since the mid 70s. They employ higher-order piece-wise polynomials which are reconstructed from the evolving cell averages 'in the direction of smoothness'. We mention here the notable examples of the high-resolution upwind FCT, MUSCL, TVD, PPM, ENO, and WENO schemes [8, 49, 19, 16, 20, 35] and this list is far from being complete. The use of intricate Riemann solvers can be avoided at the expense of using the more diffusive LxF scheme. The excessive numerical dissipation can be reduced significantly, however, when higher order piece-wise polynomial reconstructions are used in conjunction with the staggered formulation of the LxF scheme. The central scheme of Nessyahu and Tadmor (NT) [40] provides such a second-order generalization of the staggered LxF scheme. It is based on the same piece-wise linear reconstructions of cell averages used with upwind schemes, yet the solution of (approximate) Riemann problems is avoided. High resolution generalizations of the NT scheme were developed since the $90 \mathrm{~s}$ as the class of central schemes in e.g. [43, 3, 22, 21, 36, 6, 25, 2, 27, 28, 32] and here too, the list is far from being complete. The relaxation scheme of Jin and Xin [23] provides another approach which leads to a staggered central stencil for solving nonlinear conservation laws.

Being free of the (eigen-structure of -) the underlying Riemann problems, central schemes provide black-box type methods for the approximate solution of nonlinear hyperbolic conservation laws and other closely related equations [5]. Essentially, one only needs to supply the flux functions. But the staggered high-order central schemes of order, say, $r>1$, still share one disadvantage with the original LxF scheme, namely, the amplitude of their numerical viscosity of order $\mathcal{O}\left((\Delta x)^{r+1} / \Delta t\right)$. It excludes the use of small time-steps, $\Delta t$, which are too small relative to the spatial grid size $\Delta x$. The problem lies with the space-time control volumes which are staggered ' $\Delta x / 2$ - away' from each other. (Similar difficulties occur with the two dimensional conservative front tracking method which was overcome by Glimm et al. in [17], using space-time cells instead of rezoning). This problem was addressed by Kurganov and Tadmor who introduced, in [28], a new type of central schemes whose numerical viscosity is independent of $\mathcal{O}(1 / \Delta t))$. This was achieved by using variable control volumes so that cells are staggered only ' $\mathcal{O}(\Delta t)$-away' from each other. The latest version of the central-upwind scheme has been recently derived in [26]. It allows implementation of central scheme with arbitrarily small time-step, and in particular, it yields a semi-discrete formulation which can be conveniently integrated by ODE solvers, e.g., the Strong Stability Preserving (SSP) Runge-Kutta methods of [45], consult e.g., [18]. Similar advantages of a semi-discrete formulation can be achieved when a local Lax-Friedrichs building block is used over non-staggered meshes, see e.g. Shu and Osher $[45,46]$ and Liu and Osher [34]. The upwind and central schemes mentioned so far, share one thing in common - they evolve one piece of information per cell, that is, the cell average. Upwind schemes use Riemann solvers while central schemes use simpler quadrature rules. For higher accuracy, they both employ piece-wise polynomial representation of the solution which is reconstructed from these cell averages.

In [37], Y. Liu introduced an alternative technique to control the numerical dissipation of central schemes. The main idea is to evolve the solution over overlapping cells. That is, two sets of cellaverages are realized over interlacing grids. The solution is then represented as a convex combination - an ' $\mathcal{O}(\Delta t)$-weighted' combination of these overlapping cell averages. The resulting scheme has a numerical viscosity which is independent of $\mathcal{O}(1 / \Delta t)$ and as such, it admits a semi-discrete formulation which can be integrated using SSP methods. The use of overlapping cells, however, is fundamentally different in that it evolves two independent quantities for each given cell, that is, the two overlapping 
sub-cell averages. The use of overlapping cells opens many new possibilities. For example, instead of the usual reconstructions such as MUSCL and (W)ENO, overlapping cells offer a more efficient approach for high-resolution: by adding the two subcell averages, we recover the evolution of a full cell average, where by taking their difference, we independently evolve an approximate slope, rather than reconstructing it from neighboring averages. This makes feasible the use of central discontinuous Galerkin (DG) approach over overlapping cell, following the series of works of Cockburn and Shu $[13,14,15]$. Thus, in particular, the use of overlapping cells yields the versatility of finite element (Galerkin) methods which can be easily formulated on general unstructured meshes with any formal order, since no reconstruction is involved. In this paper, we further develop the staggered central DG method introduced in [38] for solving hyperbolic conservation laws. Numerical tests are performed up to third order accuracy on uniform staggered meshes in one and two dimensions. Stability analysis and error estimates, and extension of the method for time dependent and steady state convectiondiffusion equations, constitute ongoing work and will be reported in the future. Here, one does not reconstruct a piecewise-polynomial representation of the solution, but rather it is part of the evolution of higher-moments. Still, a nonlinear limiting procedure is necessary to reduce spurious oscillations for high order methods. We introduce here such a general non-oscillatory procedure, so-called hierarchical reconstruction interesting for its own sake, which is closely related to the moment limiters of Biswas et al. [7] and to the earlier work of Cockburn and Shu [13]. Since this limiting procedure requires only linear reconstructions using information from adjacent cells without characteristic decomposition, it can be easily implemented for any shapes of the cells and hence is practical also for unstructured meshes or even dynamically moving meshes (e.g. Tang and Tang [47]), although we do not pursue it in this paper. We refer the reader to [3] and the reference therein for a systematic study of central schemes on unstructured grids using the framework of discontinuous finite elements.

This paper is organized as follows. In Sec. 2, we briefly describe the central finite volume scheme on overlapping cells as the background. The natural extension to central DG scheme on overlapping cells is discussed in Sec. 3. In subsection 3.1 we study the numerical convergence rate for a number of linear and nonlinear equations having smooth solutions. In Sec. 4, we introduce a general non-oscillatory hierarchical reconstruction procedure and use it as a limiter for the central DG scheme on overlapping cells to control spurious oscillations in the presence of shocks. Numerical results testing the accuracy of the proposed schemes are included in Sections 3 and 4. Additional numerical results are presented in Sec. 5. Concluding remarks and a plan for future work are included in Sec. 6.

\section{Central Schemes on Overlapping Cells}

Consider the scalar one dimensional conservation law

$$
\frac{\partial u}{\partial t}+\frac{\partial f(u)}{\partial x}=0, \quad(x, t) \in \mathcal{R} \times(0, T)
$$

Set $\left\{x_{i}:=x_{0}+i \Delta x\right\}$, let $C_{i+1 / 2}:=\left[x_{i}, x_{i+1}\right)$ be a uniform partition of $\mathcal{R}$ and let $\left\{\bar{U}_{i+1 / 2}^{n}\right\}$ denote the set of approximate cell averages $\bar{U}_{i+1 / 2}^{n} \approx(1 / \Delta x) \int_{C_{i+1 / 2}} u\left(x, t^{n}\right) d x$. Similarly, we set $D_{i}:=$ $\left[x_{i-1 / 2}, x_{i+1 / 2}\right)$ as the dual partition and let $\left\{\bar{V}_{i}^{n}\right\}$ denote the corresponding set of approximate cell average $\bar{V}_{i}^{n} \approx(1 / \Delta x) \int_{D_{i}} u\left(x, t^{n}\right) d x$. Starting with these two piecewise-constant approximation ${ }^{1}$,

$$
\sum_{i} \bar{U}_{i+1 / 2}^{n} \mathbf{1}_{C_{i+1 / 2}}(x) \text { and } \sum_{i} \bar{V}_{i}^{n} \mathbf{1}_{D_{i}}(x)
$$

\footnotetext{
${ }^{1}$ Here and below, $\mathbf{1}_{\Omega}(x)$ denotes the characteristic function of $\Omega$
} 
we proceed to compute our approximate solution at the next time level, $t^{n+1}:=t^{n}+\Delta t^{n}$. To this end, we reconstruct two higher-order non-oscillatory piecewise-polynomial approximations,

$$
U^{n}(x)=\sum_{i} U_{i+1 / 2}(x) \mathbf{1}_{C_{i+1 / 2}}(x) \quad \text { and } \quad V^{n}(x)=\sum_{i} V_{i}(x) \mathbf{1}_{D_{i}}(x)
$$

with breakpoints at $x_{i}, i=0, \pm 1, \pm 2, \cdots$, and respectively, at $x_{i+1 / 2}, i=0, \pm 1, \pm 2, \cdots$. These piecewise-polynomials should be conservative in the sense that $\int_{C_{j+1 / 2}} U^{n}(x) d x=\Delta x \bar{U}_{j+1 / 2}^{n}$ and $\int_{D_{j}} V^{n}(x) d x=\Delta x \bar{V}_{j}^{n}$ for all $j$ 's. There are large libraries for such conservative, accurate and nonoscillatory reconstructions; we refer, for example, to the second-order example of MUSCL [48], the third-order example of [36], the well-known class of high-order (W)ENO reconstructions [20, 44], etc. Following Nessyahu and Tadmor [40], the central scheme associated with these piecewise-polynomials reads

$$
\begin{aligned}
\bar{V}_{i}^{n+1} & =\frac{1}{\Delta x} \int_{D_{i}} U^{n}(x) d x-\frac{\Delta t^{n}}{\Delta x}\left[f\left(U^{n+\frac{1}{2}}\left(x_{i+1 / 2}\right)\right)-f\left(U^{n+\frac{1}{2}}\left(x_{i-1 / 2}\right)\right)\right] \\
\bar{U}_{i+1 / 2}^{n+1} & =\frac{1}{\Delta x} \int_{C_{i+1 / 2}} V^{n}(x) d x-\frac{\Delta t^{n}}{\Delta x}\left[f\left(V^{n+\frac{1}{2}}\left(x_{i+1}\right)\right)-f\left(V^{n+\frac{1}{2}}\left(x_{i}\right)\right)\right] .
\end{aligned}
$$

To guarantee second-order accuracy, the right-hand-sides of $(2.2 \mathrm{a}),(2.2 \mathrm{~b})$ require the approximate values of $U^{n+\frac{1}{2}}\left(x_{j+1 / 2}\right) \approx u\left(x_{j+1 / 2}, t^{n+\frac{1}{2}}\right)$ and $V^{n+\frac{1}{2}}\left(x_{j}\right) \approx u\left(x_{j}, t^{n+\frac{1}{2}}\right)$ to be evaluated at the midpoint $t+\Delta t^{n} / 2$. Replacing the midpoint rule with higher order quadratures, yields higher order accuracy, e.g., $[36,6]$.

The central Nessyahu-Tadmor (NT) scheme (2.2) and its higher-order generalizations provide effective high-resolution "black-box" solvers to a wide variety of nonlinear conservation laws. When $\Delta t$ is very small, however, e.g., with $\Delta t=\mathcal{O}\left((\Delta x)^{2}\right)$ as required by the CFL condition for convectiondiffusion equations for example, the numerical dissipation of the NT schemes becomes excessively large. The excessive dissipation is due to the staggered grids where at each time-step, cell averages are shifted $\Delta x / 2$-away from each other: indeed, at the extreme of $f(u) \equiv 0$, the central scheme $(2.2)$ is reduced to re-averaging at every time step. To address this difficulty, Kurganov and Tadmor, [28], suggested to remove this excessive dissipation by using staggered grids which are shifted only $\mathcal{O}(\Delta t)-$ away from each other. This amounts to using control volumes of width $\mathcal{O}(\Delta t)$ so that the resulting schemes admits semi-discrete limit as $\Delta t \rightarrow 0$, the so called "central-upwind" schemes introduced in [28] and further generalized in [27]. Liu [37] introduced another modification of the NT scheme which removes its $\mathcal{O}(1 / \Delta t)$ dependency of numerical dissipation. In this approach, one takes advantage of the redundant representation of the solution over overlapping cells, $\bar{V}_{i}^{n}$ and $\bar{U}_{i+1 / 2}^{n}$. The idea is to use a $\mathcal{O}(\Delta t)$-dependent weighted average of $\bar{U}_{i+1 / 2}^{n}$ and $\bar{V}_{i}^{n}$. In fact the difference between them is the local dissipation error. To simplify our discussion, we momentarily give up on second-order accuracy in time, setting $U^{n+\frac{1}{2}}=U^{n}$ and $V^{n+\frac{1}{2}}=V^{n}$ in (2.2a) and (2.2b). The resulting first-order forward-Euler formulation of the new central scheme, consult figure 1, reads

$$
\begin{aligned}
\bar{V}_{i}^{n+1} & =\theta\left(\frac{1}{\Delta x} \int_{D_{i}} U^{n}(x) d x\right)+(1-\theta) \bar{V}_{i}^{n}-\frac{\Delta t^{n}}{\Delta x}\left[f\left(U^{n}\left(x_{i+1 / 2}\right)\right)-f\left(U^{n}\left(x_{i-1 / 2}\right)\right)\right] \\
\bar{U}_{i+1 / 2}^{n+1} & =\theta\left(\frac{1}{\Delta x} \int_{C_{i+1 / 2}} V^{n}(x) d x\right)+(1-\theta) \bar{U}_{i+1 / 2}^{n}-\frac{\Delta t^{n}}{\Delta x}\left[f\left(V^{n}\left(x_{i+1}\right)\right)-f\left(V^{n}\left(x_{i}\right)\right)\right]
\end{aligned}
$$

Here $\theta:=\Delta t^{n} / \Delta \tau^{n}$ where $\Delta \tau^{n}$ is an upper bound for the time step, dictated by the CFL condition. We refer the readers to [40] and [37] for more details to facilitate the full understanding of the sketches in figure 1 . Note that when $\theta=1,(2.3 \mathrm{a}),(2.3 \mathrm{~b})$ is reduced to the first-order, forward-Euler-based 


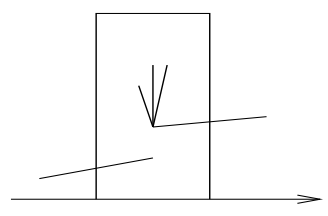

A

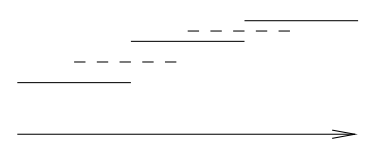

B

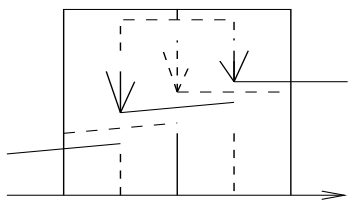

$\mathrm{C}$

Figure 1: (A) NT scheme; (B) 1D overlapping cells; (C) overlapping cells create self similarity for the grid over time and allow a convex combination of the overlapping cell averages to control the numerical dissipation.

version of the NT scheme (2.2a), (2.2b). Moreover, writing

$$
\theta\left(\frac{1}{\Delta x} \int_{D_{i}} U^{n}(x) d x\right)+(1-\theta) \bar{V}_{i}^{n}=\bar{V}_{i}^{n}+\frac{\Delta t^{n}}{\Delta \tau^{n}}\left(\frac{1}{\Delta x} \int_{D_{i}} U^{n}(x) d x-\bar{V}_{i}^{n}\right)
$$

and recalling that $\Delta \tau^{n}=\mathcal{O}(\Delta x)$ due to the CFL restriction, it follows that the local dissipative error now has a pre-factor of order, $\Delta t^{n}$, and hence the cumulative error will be independent of $\mathcal{O}(\Delta t)$. The reduced dissipation allows us to pass to a semi-discrete formulation: subtracting $\bar{V}_{i}^{n}$ and $\bar{U}_{i+1 / 2}^{n}$ from both sides, multiplying by $\frac{1}{\Delta t^{n}}$, and then passing to the limit as $\Delta t^{n} \rightarrow 0$ we end up with

$$
\frac{d}{d t} \bar{V}_{i}\left(t^{n}\right)=\frac{1}{\Delta \tau^{n}}\left(\frac{1}{\Delta x} \int_{D_{i}} U^{n}(x) d x-\bar{V}_{i}^{n}\right)-\frac{1}{\Delta x}\left[f\left(U^{n}\left(x_{i+1 / 2}\right)\right)-f\left(U^{n}\left(x_{i-1 / 2}\right)\right)\right]
$$

$(2.4 \mathrm{~b}) \frac{d}{d t} \bar{U}_{i+1 / 2}\left(t^{n}\right)=\frac{1}{\Delta \tau^{n}}\left(\frac{1}{\Delta x} \int_{C_{i+1 / 2}} V^{n}(x) d x-\bar{U}_{i+1 / 2}^{n}\right)-\frac{1}{\Delta x}\left[f\left(V^{n}\left(x_{i+1}\right)\right)-f\left(V^{n}\left(x_{i}\right)\right)\right]$.

The spatial accuracy of the semi-discrete central scheme (2.4) is dictated by the order the reconstruction $U^{n}(x)$ and $V^{n}(x)$. The SSP Runge-Kutta methods yield the matching high-order discretization in time.

We conclude this section by quoting [37] regarding the non-oscillatory behavior of the central scheme (2.4), which is quantified here in terms of the Total-Variation-Diminishing (TVD) property, e.g., [19].

Theorem 1. Consider the central schemes (2.2) and (2.3) which are set with the same initial values $\bar{V}_{i}^{n}$ and $\bar{U}_{i+1 / 2}^{n}$ at $t=t^{n}$. If the NT scheme is TVD, then so is the central scheme (2.3).

There are two reconstruction procedures for overlapping cells: one is the standard procedure to reconstruct the two classes of cell averages $\left\{\bar{V}_{i}^{n}: i=0, \pm 1, \pm 2, \cdots\right\}$ and $\left\{\bar{U}_{i+1 / 2}^{n}: i=0, \pm 1, \pm 2, \cdots\right\}$; the other couples these two classes for reconstruction of the final representation of the solution. Thus, this approach is redundant. At the same time, numerical examples in [37] have shown that by coupling the reconstructions, redundancy does provide improved resolution when compared with the one-cell average evolution approach of Godunov-type schemes.

\section{A Central Discontinuous Galerkin Method on Overlapping Cells for Conservation LaWs}

Following the general strategy of the discontinuous Galerkin (DG) methods, see e.g. Lesaint and Raviart ([31]), Cockburn ([10]), Cockburn and Shu ([13, 15]) etc., the central-type discontinuous Galerkin (DG) method on overlapping cells can be derived [38]. Consider the system of conservation laws

$$
\frac{\partial u_{k}}{\partial t}+\nabla_{\mathbf{x}} \cdot \mathbf{f}_{k}(\mathbf{u})=0, \quad(\mathbf{x}, t) \in \mathcal{R}^{d} \times(0, T), \quad k=1, \ldots, m
$$




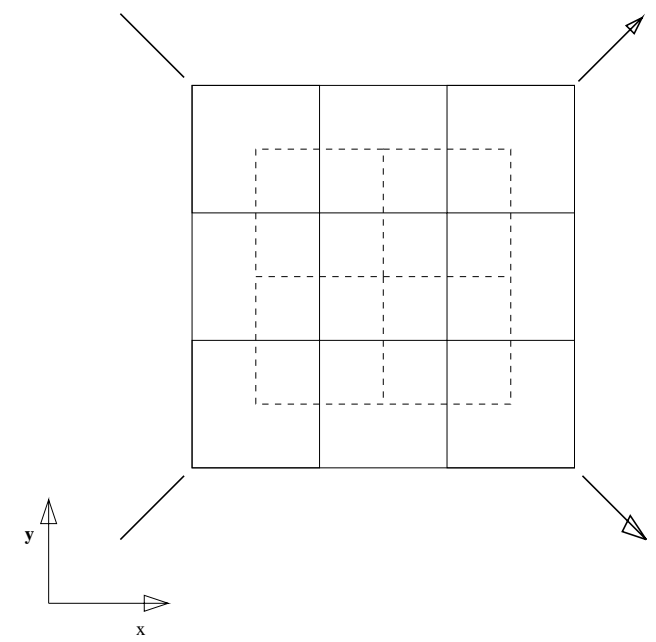

Figure 2: 2D overlapping cells by collapsing the staggered dual cells on two adjacent time levels to one time level.

where $\mathbf{u}=\left(u_{1}, \ldots, u_{m}\right)^{\top}$. For simplicity, assume a uniform staggered rectangular mesh depicted in figure 2 for the $2 \mathrm{D}$ case, and we note that the similar formulation for irregular staggered meshes, e.g., the Voronoi mesh consisting of a triangular mesh and its dual.

Let $\left\{C_{I+1 / 2}\right\}, I=\left(i_{1}, i_{2}, \cdots, i_{d}\right)$ be a partition of $R^{d}$ into uniform square cells depicted by solid lines in figure 2 and tagged by their cell centroids at the half integers, $\mathbf{x}_{I+1 / 2}:=(I+1 / 2) \Delta x$. Let $\mathcal{M}$ denote the set of piece-wise polynomials of degree $r$ on the cells $\left\{C_{I+1 / 2}\right\}$; no continuity is assumed across cell boundaries. Let $\left\{D_{I}\right\}$ be the dual mesh which consists of a $\Delta x / 2$ - shift of the $C_{I+1 / 2}$ 's depicted by dash lines in figure 2 . Let $\mathbf{x}_{I}$ be the cell centroid of the cell $D_{I}$ and let $\mathcal{N}$ denote the set of piece-wise polynomials of degree $r$ over the cells $\left\{D_{I}\right\}$; again, no continuity is assumed across the cell boundary. The weak formulation of (3.1) over these cells reads

$$
\begin{aligned}
\frac{d}{d t} \int_{C_{I+1 / 2}} u_{k} \phi d \mathbf{x} & =\int_{C_{I+1 / 2}} \mathbf{f}_{k} \cdot \nabla_{\mathbf{x}} \phi d \mathbf{x}-\int_{\partial C_{I+1 / 2}}\left(\mathbf{f}_{k} \cdot \mathbf{n}\right) \phi d s, \quad \forall \phi \in \mathcal{M}, \quad k=1, \cdots, m, \\
\frac{d}{d t} \int_{D_{I}} u_{k} \psi d \mathbf{x} & =\int_{D_{I}} \mathbf{f}_{k} \cdot \nabla_{\mathbf{x}} \psi d \mathbf{x}-\int_{\partial D_{I}}\left(\mathbf{f}_{k} \cdot \mathbf{n}\right) \psi d s, \quad \forall \psi \in \mathcal{N}, \quad k=1, \cdots, m,
\end{aligned}
$$

where $\mathbf{n}$ is the unit outer normal of the corresponding cell and $\phi$ and $\psi$ are test functions. As in the one-dimensional setup, we let

$$
\mathbf{U}^{n}(\mathbf{x})=\sum_{I+1 / 2} \mathbf{U}_{I+1 / 2}^{n}(\mathbf{x}) \mathbf{1}_{C_{I+1 / 2}}(\mathbf{x}) \in \mathcal{M} \quad \text { and } \quad \mathbf{V}^{n}(\mathbf{x})=\sum_{I} \mathbf{V}_{I}^{n}(\mathbf{x}) \mathbf{1}_{D_{I}}(\mathbf{x}) \in \mathcal{N}
$$

denote two representations of the numerical solution, approximating $\mathbf{u}\left(\cdot, t^{n}\right)$ over the two overlapping grids, $\left\{C_{I+1 / 2}\right\}$ and $\left\{D_{I}\right\}$. Observe that each of the two vector functions, $\mathbf{U}^{n}$ with smooth pieces supported on the $C_{I+1 / 2}$ 's and $\mathbf{V}^{n}$ with smooth pieces supported on the $D_{I}$ 's, consist of $m$ components

$$
\mathbf{U}^{n}(\mathbf{x})=\left(U_{1}^{n}(\mathbf{x}), \ldots, U_{m}^{n}(\mathbf{x})\right)^{\top} \text { and } \mathbf{V}^{n}(\mathbf{x})=\left(V_{1}^{n}(\mathbf{x}), \ldots, V_{m}^{n}(\mathbf{x})\right)^{\top} .
$$

Given these conservative, accurate and non-oscillatory approximations at $t^{n}$ we proceed to compute the approximate solution at the next time level, $t^{n+1}=t^{n}+\Delta t^{n}$. To this end, the exact solution $\mathbf{u}\left(\mathbf{x}, t^{n}\right)$ of (3.1) in the right-hand-side of $(3.2 \mathrm{a})$, is replaced by $\mathbf{V}^{n}(\mathbf{x})=\left(V_{1}^{n}, \ldots, V_{m}^{n}\right)^{\top}$; similarly, for the right-hand-side of $(3.2 \mathrm{~b})$ we use the approximate solution $\mathbf{U}^{n}(\mathbf{x})=\left(U_{1}^{n}, \ldots, U_{m}^{n}\right)^{\top}$. Further, time derivatives on the left are replaced by Forward Euler time differencing where we use the same type of $\theta$-weighting of the $\mathbf{U}^{n}$ 's and the $\mathbf{V}^{n}$ 's as in (2.3a), (2.3b). In the resulting central discontinuous 
Galerkin method one seeks piece-wise polynomials $\left\{\mathbf{U}_{I+1 / 2}^{n+1}\right\} \in \mathcal{M}$ and $\left\{\mathbf{V}_{I}^{n+1}\right\} \in \mathcal{N}$ such that for all I's,

$$
\int_{C_{I+1 / 2}} U_{k}^{n+1}(\mathbf{x}) \phi(\mathbf{x}) d \mathbf{x}=\theta \int_{C_{I+1 / 2}} V_{k}^{n}(\mathbf{x}) \phi(\mathbf{x}) d \mathbf{x}+(1-\theta) \int_{C_{I+1 / 2}} U_{k}^{n}(\mathbf{x}) \phi(\mathbf{x}) d \mathbf{x}
$$

$$
\begin{aligned}
& +\Delta t^{n} \int_{C_{I+1 / 2}} \mathbf{f}_{k}\left(\mathbf{V}^{n}(\mathbf{x})\right) \cdot \nabla_{\mathbf{x}} \phi d \mathbf{x} \\
& -\Delta t^{n} \int_{\partial C_{I+1 / 2}}\left(\mathbf{f}_{k}\left(\mathbf{V}^{n}(\mathbf{x})\right) \cdot \mathbf{n}\right) \phi(\mathbf{x}) d s, \quad \forall \phi \in \mathcal{M}, k=1, \cdots, m, \\
& \int_{D_{I}} V_{k}^{n+1}(\mathbf{x}) \psi(\mathbf{x}) d \mathbf{x}=\theta \int_{D_{I}} U_{k}^{n}(\mathbf{x}) \psi(\mathbf{x}) d \mathbf{x}+(1-\theta) \int_{D_{I}} V_{k}^{n}(\mathbf{x}) \psi(\mathbf{x}) d \mathbf{x} \\
& +\Delta t^{n} \int_{D_{I}} \mathbf{f}_{k}\left(\mathbf{U}^{n}(\mathbf{x})\right) \cdot \nabla_{\mathbf{x}} \psi(\mathbf{x}) d \mathbf{x} \\
& -\Delta t^{n} \int_{\partial D_{I}}\left(\mathbf{f}_{k}\left(\mathbf{U}^{n}(\mathbf{x})\right) \cdot \mathbf{n}\right) \psi(\mathbf{x}) d s, \quad \forall \psi \in \mathcal{N}, k=1, \cdots, m
\end{aligned}
$$

Here $\theta=\Delta t^{n} / \Delta \tau^{n} \leq 1, \Delta \tau^{n}$ is the maximum time step size determined by the CFL restriction, $\Delta t^{n}=$ $t^{n+1}-t^{n}$ is the current time step size. $\Delta \tau^{n}=$ (CFL factor $) \times \Delta x /($ maximum characteristic speed $)$, where the CFL factor should be less than 1/2. At the time $t^{n}, \Delta \tau^{n}$ is first chosen with certain CFL factor, then $\Delta t^{n}$ has the freedom to take any value in $\left(0, \Delta \tau^{n}\right]$ without introducing excessive dissipation. The smaller $\Delta \tau^{n}$ is chosen, the larger the numerical dissipation is. We find in numerical experiments that setting $\Delta \tau^{n}$ with CFL factor 0.4 is robust. In some numerical tests with less interactions of discontinuities, we can choose larger $\Delta \tau^{n}$. This forward Euler step is going to be used in a SSP Runge-Kutta method of desired order. For the pure hyperbolic problem, $\Delta t^{n}$ can be chosen as large as possible, i.e., $\Delta t^{n}=\Delta \tau^{n}$ for efficiency.

The resulting central DG is the two-dimensional analogue of the one-dimensional central scheme (2.4). And as in the one-dimensional case, semi-discrete version of (3.3) can be obtained; higher order Runge-Kutta time discretization can be used to match the high-order accuracy of the spatial reconstructions. We conclude with the semi-discrete central DG approximation of (3.1) such that for all admissible test functions $\phi$ and $\psi$ and all $I$ 's, 


$$
\begin{aligned}
\frac{d}{d t} \int_{C_{I+1 / 2}} U_{k} \phi d \mathbf{x}= & \frac{1}{\Delta \tau} \int_{C_{I+1 / 2}}\left(V_{k}(\mathbf{x})-U_{k}(\mathbf{x})\right) \phi(\mathbf{x}) d \mathbf{x} \\
& +\int_{C_{I+1 / 2}} \mathbf{f}_{k}(\mathbf{V}(\mathbf{x})) \cdot \nabla_{\mathbf{x}} \phi d \mathbf{x} \\
& -\int_{\partial C_{I+1 / 2}}\left(\mathbf{f}_{k}(\mathbf{V}(\mathbf{x})) \cdot \mathbf{n}\right) \phi(\mathbf{x}) d s, \quad \forall \phi \in \mathcal{M}, k=1, \cdots, m \\
\frac{d}{d t} \int_{D_{I}} V_{k} \psi d \mathbf{x}= & \frac{1}{\Delta \tau} \int_{D_{I}}\left(U_{k}(\mathbf{x})-V_{k}(\mathbf{x})\right) \psi(\mathbf{x}) d \mathbf{x} \\
& +\int_{D_{I}} \mathbf{f}_{k}(\mathbf{U}(\mathbf{x})) \cdot \nabla_{\mathbf{x}} \psi(\mathbf{x}) d \mathbf{x} \\
& -\int_{\partial D_{I}}\left(\mathbf{f}_{k}(\mathbf{U}(\mathbf{x})) \cdot \mathbf{n}\right) \psi(\mathbf{x}) d s, \quad \forall \psi \in \mathcal{N}, k=1, \cdots, m .
\end{aligned}
$$

For example, consider the piece-wise quadratic element in $2 \mathrm{D}$, see e.g. figure 2 . We use the third order SSP Runge-Kutta method [45] to discretize (3.4) in time, which ends up with calling the forward Euler step (3.3) three times. Let cell $C_{I+1 / 2}$ as in (3.3a) be the cell bounded by solid lines in the center of figure 2, and let

$$
\begin{aligned}
U_{I+1 / 2}\left(x-x_{I+1 / 2}, y-y_{I+1 / 2}\right)= & U_{I+1 / 2}(0,0)+\partial_{x} U_{I+1 / 2}(0,0)\left(x-x_{I+1 / 2}\right)+ \\
& \partial_{y} U_{I+1 / 2}(0,0)\left(y-y_{I+1 / 2}\right)+\frac{1}{2} \partial_{x x} U_{I+1 / 2}(0,0)\left(x-x_{I+1 / 2}\right)^{2}+ \\
& \partial_{x y} U_{I+1 / 2}(0,0)\left(x-x_{I+1 / 2}\right)\left(y-y_{I+1 / 2}\right)+ \\
& \frac{1}{2} \partial_{y y} U_{I+1 / 2}(0,0)\left(y-y_{I+1 / 2}\right)^{2}
\end{aligned}
$$

be $\left.U_{k}^{n+1}\right|_{C_{I+1 / 2}}$, i.e., $U_{k}^{n+1}(\mathbf{x})$ restricted in cell $C_{I+1 / 2}$, where $\left(x_{I+1 / 2}, y_{I+1 / 2}\right)$ is the cell centroid of cell $C_{I+1 / 2}$. There are 6 coefficients to be determined in this polynomial in cell $C_{I+1 / 2}$, namely

By letting

$$
\begin{gathered}
U_{I+1 / 2}(0,0), \partial_{x} U_{I+1 / 2}(0,0), \partial_{y} U_{I+1 / 2}(0,0), \\
\frac{1}{2} \partial_{x x} U_{I+1 / 2}(0,0), \partial_{x y} U_{I+1 / 2}(0,0), \frac{1}{2} \partial_{y y} U_{I+1 / 2}(0,0) .
\end{gathered}
$$

$$
\phi(\mathbf{x})=1, x-x_{I+1 / 2}, y-y_{I+1 / 2},\left(x-x_{I+1 / 2}\right)^{2},\left(x-x_{I+1 / 2}\right)\left(y-y_{I+1 / 2}\right) \text { or }\left(y-y_{I+1 / 2}\right)^{2},
$$

we obtain 6 linear equations in (3.3a) to solve for $U_{I+1 / 2}\left(x-x_{I+1 / 2}, y-y_{I+1 / 2}\right)$. The last two integrals in (3.3a) can be approximated by Gaussian quadratures, such as the 3-point Gaussian quadrature for line integrals. The other integrals on the right hand side of (3.3a) can be evaluated exactly.

3.1. Numerical Errors for Smooth Solutions. In this subsection we study the convergence rate for a number of equations having smooth solutions. The examples are computed by linear schemes described previously without using any limiter.

Example 1. Let us start with the following linear transport equation with periodic boundary conditions

$$
\begin{aligned}
u_{t}+a u_{x}=0, & (x, t) \in(0,2) \times(0,2), \\
u(x, 0)=1+\sin (\pi x), & x \in(0,2),
\end{aligned}
$$


TABLE 1. $P^{1}$ version of the central DG scheme (3.4) for the linear convection equation $(3.5)$.

\begin{tabular}{|c|c|c|c|c|c|}
\hline$\Delta x$ & $1 / 20$ & $1 / 40$ & $1 / 80$ & $1 / 160$ & $1 / 320$ \\
\hline$L_{1}$ error & $8.91 \mathrm{E}-3$ & $2.25 \mathrm{E}-3$ & $5.66 \mathrm{E}-4$ & $1.42 \mathrm{E}-4$ & $3.54 \mathrm{E}-5$ \\
\hline order & - & 1.99 & 1.99 & 1.99 & 2.00 \\
\hline$L_{\infty}$ error & $5.92 \mathrm{E}-3$ & $1.55 \mathrm{E}-3$ & $3.96 \mathrm{E}-4$ & $1.00 \mathrm{E}-4$ & $2.51 \mathrm{E}-5$ \\
\hline order & - & 1.93 & 1.97 & 1.99 & 1.99 \\
\hline
\end{tabular}

\begin{tabular}{|c|c|c|c|c|c|}
\hline$\Delta x$ & $1 / 2$ & $1 / 4$ & $1 / 8$ & $1 / 16$ & $1 / 32$ \\
\hline$L_{1}$ error & $6.69 \mathrm{E}-2$ & $3.29 \mathrm{E}-2$ & $5.04 \mathrm{E}-3$ & $1.66 \mathrm{E}-3$ & $3.88 \mathrm{E}-4$ \\
\hline order & - & 1.02 & 2.70 & 1.60 & 2.10 \\
\hline$L_{\infty}$ error & $3.85 \mathrm{E}-2$ & $2.05 \mathrm{E}-2$ & $7.69 \mathrm{E}-3$ & $1.19 \mathrm{E}-3$ & $2.75 \mathrm{E}-4$ \\
\hline order & - & 0.91 & 1.41 & 2.69 & 2.11 \\
\hline
\end{tabular}

TABle 2. $P^{1}$ version of the central DG scheme (3.4) for the 2D Burgers' equation.

\begin{tabular}{|c|c|c|c|c|c|}
\hline$\Delta x$ & $1 / 20$ & $1 / 40$ & $1 / 80$ & $1 / 160$ & $1 / 320$ \\
\hline$L_{1}$ error & $6.50 \mathrm{E}-5$ & $8.12 \mathrm{E}-6$ & $1.02 \mathrm{E}-6$ & $1.27 \mathrm{E}-7$ & $1.59 \mathrm{E}-8$ \\
\hline order & - & 3.00 & 2.99 & 3.01 & 3.00 \\
\hline$L_{\infty}$ error & $4.68 \mathrm{E}-5$ & $5.90 \mathrm{E}-6$ & $7.40 \mathrm{E}-7$ & $9.27 \mathrm{E}-8$ & $1.16 \mathrm{E}-8$ \\
\hline order & - & 2.99 & 3.00 & 3.00 & 3.00 \\
\hline
\end{tabular}

TABle 3. $P^{2}$ version of the central DG scheme (3.4) for the linear convection equation $(3.5)$.

\begin{tabular}{|c|c|c|c|c|}
\hline$\Delta x$ & $1 / 20$ & $1 / 40$ & $1 / 80$ & $1 / 160$ \\
\hline$L_{\infty}$ error & $9.32 \mathrm{E}-7$ & $5.89 \mathrm{E}-8$ & $3.70 \mathrm{E}-9$ & $2.32 \mathrm{E}-10$ \\
\hline order & - & 3.98 & 3.99 & 4.00 \\
\hline$L_{\infty}$ error,$\Delta t=\Delta x^{2}$ & $9.32 \mathrm{E}-7$ & $5.89 \mathrm{E}-8$ & $3.70 \mathrm{E}-9$ & $2.32 \mathrm{E}-10$ \\
\hline order & - & 3.98 & 3.99 & 4.00 \\
\hline
\end{tabular}

TABle 4. $P^{2}$ version of the central DG scheme (3.4) for equation (3.5) with $a=0$.

where $a=1$ by default.

The test results at $T=2$ for the $P^{1}$ (piece-wise linear) version of the central DG scheme on overlapping cells (3.4) are listed in Table 1, with second order Runge-Kutta time discretization. The CFL factor is 0.4 for choosing $\Delta \tau$ and the actual time step size $\Delta t$ is chosen with $\theta=0.9$. It can be seen that the expected second order accuracy is achieved. The results for the $P^{2}$ (piece-wise quadratic) version of the scheme (3.4) for the linear convection equation (3.5) are listed in Table 3, with a third order TVD Runge-Kutta time discretization [45]. The results for the same equation with $a=0$ are listed in Table 4, in which the first row is computed with the previously chosen $\Delta t$ and the second row is computed with $\Delta t=\Delta x^{2}$. We observe that the staggered dissipation error does not increase with a diminishing time step size. We remark that for this special case with $a=0$, the $\Delta \tau$ can be chosen as $+\infty$, since there is no CFL restriction on the stability time step. With this choice of $\Delta \tau$, our scheme will maintain exactly the initial condition for this degenerated PDE. If we choose a finite $\Delta \tau^{n}$ anyway, then the initial solution may not be maintained exactly. As to the order of accuracy, 


\begin{tabular}{|c|c|c|c|c|c|}
\hline$\Delta x$ & $1 / 10$ & $1 / 20$ & $1 / 40$ & $1 / 80$ & $1 / 160$ \\
\hline$L_{1}$ error & $2.72 \mathrm{E}-5$ & $3.41 \mathrm{E}-6$ & $4.29 \mathrm{E}-7$ & $5.37 \mathrm{E}-8$ & $6.78 \mathrm{E}-9$ \\
\hline order & - & 3.00 & 2.99 & 3.00 & 2.99 \\
\hline$L_{\infty}$ error & $4.00 \mathrm{E}-5$ & $7.06 \mathrm{E}-6$ & $8.27 \mathrm{E}-7$ & $1.04 \mathrm{E}-7$ & $1.31 \mathrm{E}-8$ \\
\hline order & - & 2.50 & 3.09 & 2.99 & 2.99 \\
\hline
\end{tabular}

TABLE 5. $P^{2}$ version of the central DG scheme (3.4) for the 1D Burgers' equation.

\begin{tabular}{|c|c|c|c|c|c|}
\hline$\Delta x$ & $1 / 4$ & $1 / 8$ & $1 / 16$ & $1 / 32$ & $1 / 64$ \\
\hline$L_{1}$ error & $8.33 \mathrm{E}-3$ & $9.58 \mathrm{E}-4$ & $1.36 \mathrm{E}-4$ & $1.65 \mathrm{E}-5$ & $2.14 \mathrm{E}-6$ \\
\hline order & - & 3.12 & 2.82 & 3.04 & 2.95 \\
\hline$L_{\infty}$ error & $4.56 \mathrm{E}-3$ & $8.20 \mathrm{E}-4$ & $1.48 \mathrm{E}-4$ & $1.95 \mathrm{E}-5$ & $2.58 \mathrm{E}-6$ \\
\hline order & - & 2.48 & 2.47 & 2.92 & 2.92 \\
\hline
\end{tabular}

TABLE 6. $P^{2}$ version of the central DG scheme (3.4) for the 2D Burgers' equation.

\begin{tabular}{|c|c|c|c|c|c|}
\hline$\Delta x$ & $1 / 4$ & $1 / 8$ & $1 / 16$ & $1 / 32$ & $1 / 64$ \\
\hline$L_{1}$ error & $5.35 \mathrm{E}-3$ & $5.75 \mathrm{E}-4$ & $6.80 \mathrm{E}-5$ & $7.81 \mathrm{E}-6$ & $9.77 \mathrm{E}-7$ \\
\hline order & - & 3.22 & 3.08 & 3.12 & 3.00 \\
\hline$L_{\infty}$ error & $2.57 \mathrm{E}-3$ & $3.16 \mathrm{E}-4$ & $8.00 \mathrm{E}-5$ & $1.10 \mathrm{E}-5$ & $1.53 \mathrm{E}-6$ \\
\hline order & - & 3.02 & 1.98 & 2.86 & 2.85 \\
\hline
\end{tabular}

TABLE 7. $P^{2}$ version of the central DG scheme (3.4) for the 2D nonlinear equation.

we can see that the expected third order accuracy is achieved in Table 3 and fourth order accuracy, which is one order higher than expected, is achieved in Table 4.

Example 2. We test the scheme for the Burgers' equation $u_{t}+\left(\frac{1}{2} u^{2}\right)_{x}=0, u(x, 0)=\frac{1}{4}+\frac{1}{2} \sin (\pi x)$. The errors are shown in Table 5 at the final time $T=0.1$ when the solution is still smooth.

Example 3. We conduct a convergence test for the $P^{1}$ version of the scheme (3.4) on a $2 \mathrm{D}$ problem [12] which is the Burgers' equation with periodic initial data: $u_{t}+\left(\frac{1}{2} u^{2}\right)_{x}+\left(\frac{1}{2} u^{2}\right)_{y}=0$ on $[-1,1] \times[-1,1], u(x, y, 0)=\frac{1}{4}+\frac{1}{2} \sin (\pi(x+y))$. The numerical solutions are computed at the final time $T=0.1$ when the exact solution is still smooth. The CFL factor is 0.4 for choosing $\Delta \tau$ and the actual time step size $\Delta t$ is chosen with $\theta=0.9$. The errors are shown in Table 2. Again we observe the expected second order accuracy. Further we test the $P^{2}$ version of scheme for the 2D Burgers' equation. The errors are shown in Table 6 at the final time $T=0.1$.

Example 4. The solution of the 2D Burgers' equation may contain linear waves, hence we also test the scheme on another $2 \mathrm{D}$ equation $u_{t}+\left(\frac{1}{2} u^{2}\right)_{x}+\left(\frac{1}{4} u^{4}\right)_{y}=0, u(x, 0)=\frac{1}{4}+\frac{1}{2} \sin (\pi(x+y))$. The accuracy of the numerical solution is shown at $T=0.1$ in Table 7 .

It seems that for all these cases the expected third order accuracy is achieved for the $P^{2}$ version of scheme, at least for the $L_{1}$ errors.

\section{A General Non-Oscillatory Hierarchical Reconstruction Procedure}

Compared to finite volume schemes which evolve only cell averages over time, discontinuous Galerkin methods compute and evolve a high order polynomial in each cell. How to take advantage of the extra information provided by the DG method in each cell and use it in the limiting process where the solution is non-smooth is a challenge. The first idea is given by Cockburn and Shu [13] for the DG method which limits the variation between a cell edge value and its cell average by the differences between the cell averages of the current and neighboring cells. The higher Legendre moments are 
truncated in a cell if non-smoothness is detected. This process is shown to be total variation bounded in the means. A generalization is introduced in Biswas et al. [7], which detects the non-smoothness in higher degree moments and applies the limiting when necessary from higher to lower moments. In Qiu and Shu [42, 41], a high order WENO reconstruction is used as a limiter for the so-called "troubled" cells, where the polynomial defined at Gaussian points is reconstructed from a WENO procedure and is projected back to the finite element space to replace the one computed by the DG method. In [41], the Hermite WENO reconstruction takes not only cell averages of a function, but also cell averages of its first order derivatives in order to obtain a compact reconstruction. A similar strategy is used in our non-oscillatory hierarchical reconstruction, where cell averages of various orders of derivatives of a function are to be calculated and used in the reconstruction of linear polynomials in each stage. Our limiting procedure is closely related to [7]. Our departure from [7] begins with a different point of view, where the approximation in each cell is viewed as a high degree polynomial, instead of the combination of orthogonal Legendre polynomials advocated in [7]. Instead of a limiting procedure which is trying to set an acceptable range for the coefficient of the Legendre moments (by using the coefficients of lower-degree moments), we reconstruct the complete set of coefficients of the $m$-degree polynomial terms, using a non-oscillatory conservative reconstruction which involves previous reconstructed terms of degrees above $m$. The resulting, so-called hierarchical reconstruction algorithm is easy to implement in a multi-dimensional setting and there is no need to transform an irregular mesh cell into a rectangular one or use a dimension-by-dimension extension of a 1D limiter. It is essentially independent of the shapes of mesh cells and is compact because of the conservative non-oscillatory linear reconstruction (such as the MUSCL or second order ENO reconstruction, see [1] for an implementation for unstructured meshes) used in each stage. We now give the details of this reconstruction procedure. For simplicity we only discuss the scalar case. For systems a componentby-component extension is applied without characteristic decomposition.

From scheme (3.4) with the SSP Runge-Kutta methods, we obtain numerical solutions $U^{n}(\mathbf{x})$ and $V^{n}(\mathbf{x})$ at the time $t^{n}$. To simplify the notation we drop the superscript $n$ and write

$$
U(\mathbf{x})=\sum_{I+1 / 2} U_{I+1 / 2}\left(\mathbf{x}-\mathbf{x}_{I+1 / 2}\right) \mathbf{1}_{C_{I+1 / 2}}(\mathbf{x}) \in \mathcal{M} \quad \text { and } \quad V(\mathbf{x})=\sum_{I} V_{I}\left(\mathbf{x}-\mathbf{x}_{I}\right) \mathbf{1}_{D_{I}}(\mathbf{x}) \in \mathcal{N},
$$

recalling that $\mathbf{x}_{I+1 / 2}$ and $\mathbf{x}_{I}$ are centroids of cell $C_{I+1 / 2}$ and $D_{I}$ respectively; $U_{I+1 / 2}\left(\mathbf{x}-\mathbf{x}_{I+1 / 2}\right)$ and $V_{I}\left(\mathbf{x}-\mathbf{x}_{I}\right)$ are the polynomials (of degree $r$ ) in cells $C_{I+1 / 2}$ and $D_{I}$ respectively ${ }^{2}$. The task is to reconstruct a 'limited' version of these polynomials, retaining high-resolution and removing spurious oscillations. In the following, we discuss a hierarchical reconstruction procedure to recompute the polynomial $U_{I+1 / 2}\left(\mathbf{x}-\mathbf{x}_{I+1 / 2}\right)$ by using polynomials in cells adjacent to cell $C_{I+1 / 2}$. For convenience these adjacent cells are renamed as the set $\left\{C_{J}\right\}$ (which contain cell $C_{I+1 / 2}, D_{I}$ etc), and the polynomials (of degree $r$ ) supported on them are thus renamed as $\left\{U_{J}\left(\mathbf{x}-\mathbf{x}_{J}\right)\right\}$ respectively, where $\mathbf{x}_{J}$ is the cell centroid of cell $C_{J}$. We write $U_{I+1 / 2}\left(\mathbf{x}-\mathbf{x}_{I+1 / 2}\right)$ in terms of its Taylor expansion,

$$
U_{I+1 / 2}\left(\mathbf{x}-\mathbf{x}_{I+1 / 2}\right)=\sum_{m=0}^{r} \sum_{|\mathbf{m}|=m} \frac{1}{\mathbf{m} !} U_{I+1 / 2}^{(\mathbf{m})}(\mathbf{0})\left(\mathbf{x}-\mathbf{x}_{I+1 / 2}\right)^{\mathbf{m}},
$$

where $\frac{1}{\mathbf{m} !} U_{I+1 / 2}^{(\mathbf{m})}(\mathbf{0})$ are the coefficients which participate in its typical $m$-degree terms,

$$
\sum_{|\mathbf{m}|=m} \frac{1}{\mathbf{m} !} U_{I+1 / 2}^{(\mathbf{m})}(\mathbf{0})\left(\mathbf{x}-\mathbf{x}_{I+1 / 2}\right)^{\mathbf{m}},|\mathbf{m}|=0, \ldots, r,
$$

\footnotetext{
${ }^{2}$ These polynomials could be oscillatory. There could be other methods to compute these polynomials such as a finite volume reconstruction from cell averages.
} 
$\mathbf{m}=\left(m_{1}, m_{2}, \cdots, m_{d}\right)$ is the multi-index,

$$
|\mathbf{m}|=\sum_{i=1}^{d} m_{i}, \mathbf{m} !=\prod_{i=1}^{d} m_{i} !, \quad U_{I+1 / 2}^{(\mathbf{m})}(\mathbf{x})=\partial_{x_{d}}^{m_{d}} \cdots \partial_{x_{1}}^{m_{1}} U_{I+1 / 2}(\mathbf{x})
$$

and $\mathbf{x}=\left(x_{1}, \cdots, x_{d}\right)$. The following hierarchical reconstruction describes a procedure to compute the new coefficients

$$
\frac{1}{\mathbf{m} !} \widetilde{U}_{I+1 / 2}^{(\mathbf{m})}(\mathbf{0}), \quad m=r, r-1, \ldots, 0
$$

in $U_{I+1 / 2}\left(\mathbf{x}-\mathbf{x}_{I+1 / 2}\right)$, iterating from the highest to the lowest degree terms.
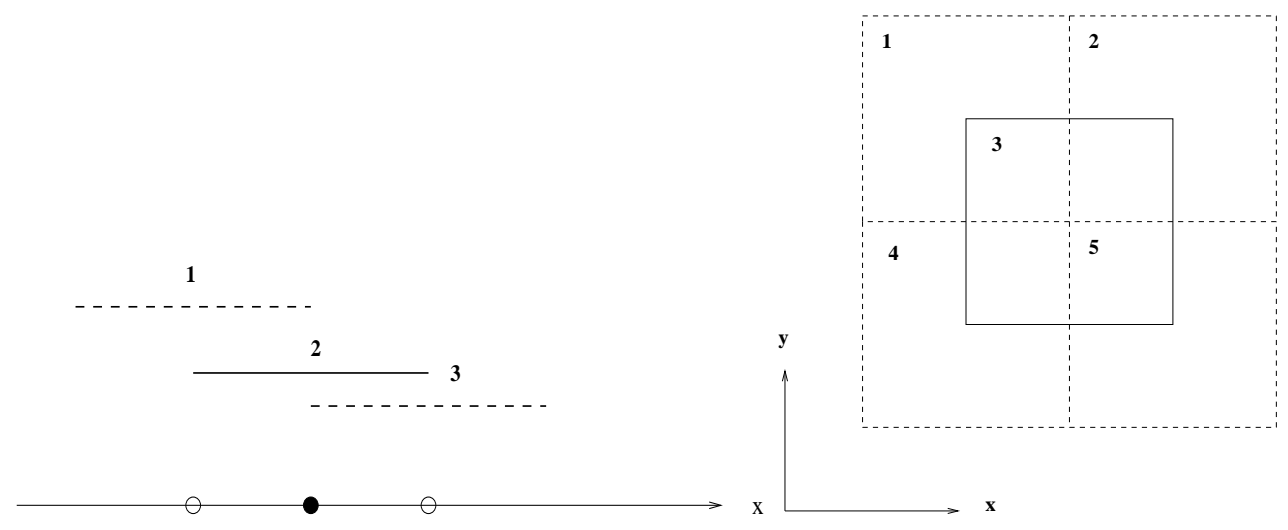

Figure 3: Left: 1D non-oscillatory hierarchical reconstruction for cell 2 involves only overlapping cells 1,2 and 3. Right: 2D non-oscillatory hierarchical reconstruction for cell 3 involves only overlapping cells $1,2,3,4$ and 5 .

4.1. An Example for Piece-wise Quadratic Finite Element Space in 1D. Suppose $U_{j}\left(x-x_{j}\right)=$ $U_{j}(0)+U_{j}^{\prime}(0)\left(x-x_{j}\right)+\frac{1}{2} U_{j}^{\prime \prime}(0)\left(x-x_{j}\right)^{2}, j=1,2,3$, are given at cells $C_{1}, C_{2}$ and $C_{3}$ respectively (see figure 3 left), where $x_{j}$ is the center of cell $C_{j}$. These polynomials could be oscillatory if located near a discontinuity of the weak solution. The following algorithm computes a new value for each coefficient in the polynomial defined on cell $C_{2}$, in order to reduce the oscillation while keeping the accuracy (in smooth area) and resolution.

Step 1. (1) Take the first derivative for them to obtain $L_{j}\left(x-x_{j}\right)=U_{j}^{\prime}(0)+U_{j}^{\prime \prime}(0)\left(x-x_{j}\right)$, $j=1,2,3$.

(2) Calculate the cell average of $L_{j}\left(x-x_{j}\right)$ on cell $C_{j}$ to obtain $\bar{L}_{j}=U_{j}^{\prime}(0), j=1,2,3$.

(3) With the three cell averages one can apply a MUSCL or second order ENO procedure to reconstruct a non-oscillatory linear polynomial in cell $C_{2}$. The slope of this new linear polynomial corresponds to the slope $U_{2}^{\prime \prime}(0)$ of the original linear polynomial $L_{2}\left(x-x_{2}\right)$ in cell $C_{2}$, and is denoted by $\widetilde{U}_{2}^{\prime \prime}(0)$. The details can be explained as follows.

Using the technique of [1], let the new linear polynomial $\widetilde{L}_{2}\left(x-x_{2}\right)$ in cell $C_{2}$ be determined by solving

$$
\frac{1}{\left|C_{j}\right|} \int_{C_{j}} \widetilde{L}_{2}\left(x-x_{2}\right) d x=\bar{L}_{j}, \quad j=1,2
$$

We now obtain the slope of $\widetilde{L}_{2}\left(x-x_{2}\right)$, which is only a candidate for the new value of $U_{2}^{\prime \prime}(0)$. The set of cells $\left\{C_{1}, C_{2}\right\}$ chosen by $C_{j}$ in (4.1) is called a stencil for cell $C_{2}$. We can similarly determine 
another candidate for the new value of $U_{2}^{\prime \prime}(0)$ by solving equation (4.1) with $C_{j}$ chosen from another stencil $\left\{C_{2}, C_{3}\right\}$ of cells. Finally we let

$$
\widetilde{U}_{2}^{\prime \prime}(0)=\operatorname{minmod}\left(\text { candidates of } U_{2}^{\prime \prime}(0)\right),
$$

where

$$
\operatorname{minmod}\left\{c_{1}, c_{2}, \cdots, c_{m}\right\}= \begin{cases}\min \left\{c_{1}, c_{2}, \cdots, c_{m}\right\}, & \text { if } c_{1}, c_{2}, \cdots, c_{m}>0, \\ \max \left\{c_{1}, c_{2}, \cdots, c_{m}\right\}, & \text { if } c_{1}, c_{2}, \cdots, c_{m}<0, \\ 0, & \text { otherwise }\end{cases}
$$

This is a MUSCL reconstruction. To use the second order ENO reconstruction, we replace the minmod function by the following $\operatorname{minmod}_{2}$ function.

$$
\operatorname{minmod}_{2}\left\{c_{1}, c_{2}, \cdots, c_{m}\right\}=c_{j}, \quad \text { if }\left|c_{j}\right|=\min \left\{\left|c_{1}\right|,\left|c_{2}\right|, \cdots,\left|c_{m}\right|\right\} .
$$

In order to find the new value $\widetilde{U}_{2}^{\prime}(0)$ for $U_{2}^{\prime}(0)$ by using the above MUSCL or second order ENO reconstruction, we need to find the cell averages of the linear part $U_{2}(0)+U_{2}^{\prime}(0)\left(x-x_{2}\right)$ on cell $C_{2}$ and its neighbors $C_{1}$ and $C_{3}$.

Step 2. (1) Compute the cell average of $U_{j}\left(x-x_{j}\right)$ on cell $C_{j}$ to obtain $\overline{U_{j}}, j=1,2,3$.

(2) Compute the cell average of the polynomial $\widetilde{R}_{2}\left(x-x_{2}\right)=\frac{1}{2} \widetilde{U}_{2}^{\prime \prime}(0)\left(x-x_{2}\right)^{2}$ on cell $C_{j}$ to obtain $\bar{R}_{j}, j=1,2,3$.

(3) Redefine $\bar{L}_{j}=\overline{U_{j}}-\bar{R}_{j}, j=1,2,3$. These are the approximate cell averages of the linear part $U_{2}(0)+U_{2}^{\prime}(0)\left(x-x_{2}\right)$ on cells $C_{1}, C_{2}$ and $C_{3}$.

(4) Similar to Step 1, we solve equation (4.1) to obtain a linear polynomial in cell $C_{2}$. The slope of this linear polynomial corresponds to the slope $U_{2}^{\prime}(0)$ of the linear polynomial $U_{2}(0)+U_{2}^{\prime}(0)\left(x-x_{2}\right)$, and is only a candidate for the new value of $U_{2}^{\prime}(0)$. Another candidate can be obtained by solving (4.1) with $C_{j}$ chosen from another stencil $\left\{C_{2}, C_{3}\right\}$ of cells. Finally let

$$
\widetilde{U}_{2}^{\prime}(0)=\operatorname{minmod}\left(\text { candidates of } U_{2}^{\prime}(0)\right) \text {. }
$$

For the second order ENO reconstruction, the minmod function can be replaced by the $\operatorname{minmod}_{2}$ function. To keep the cell average invariant, we let the new value for $U_{2}(0)$ be $\widetilde{U}_{2}(0)=\bar{L}_{2}$.

The convergence test results with Algorithm 1 for Example 2 can be found in Tables 8 and 9. We observe that the order of accuracy is maintained, although (as expected for any limiter) the magnitude of the error is increased for the same mesh (see Table 5 for a comparison).

4.2. Hierarchical Reconstruction - General Description. In the following, we discuss a hierarchical reconstruction procedure to recompute the polynomial $U_{I+1 / 2}\left(\mathbf{x}-\mathbf{x}_{I+1 / 2}\right)$ by using polynomials in cells adjacent to cell $C_{I+1 / 2}$. Recall that these adjacent cells are renamed as the set $\left\{C_{J}\right\}$ and the polynomials (of degree $r$ ) supported on them are thus renamed as $\left\{U_{J}\left(\mathbf{x}-\mathbf{x}_{J}\right)\right\}$ respectively. The following hierarchical reconstruction describes a procedure to compute the new coefficients

$$
\frac{1}{\mathbf{m} !} \widetilde{U}_{I+1 / 2}^{(\mathbf{m})}(\mathbf{0}), \quad m=r, r-1, \ldots, 0
$$

in $U_{I+1 / 2}\left(\mathbf{x}-\mathbf{x}_{I+1 / 2}\right)$, iterating from the highest to the lowest degree terms.

To reconstruct $\widetilde{U}_{I+1 / 2}^{(\mathbf{m})}(\mathbf{0})$, we first compute many candidates of $U_{I+1 / 2}^{(\mathbf{m})}(\mathbf{0})$ (sometimes still denoted as $\widetilde{U}_{I+1 / 2}^{(\mathbf{m})}(\mathbf{0})$ with specification), and we then let the new coefficient for $U_{I+1 / 2}^{(\mathbf{m})}(\mathbf{0})$ be

$$
\widetilde{U}_{I+1 / 2}^{(\mathbf{m})}(\mathbf{0})=F\left(\text { candidates of } U_{I+1 / 2}^{(\mathbf{m})}(\mathbf{0})\right),
$$

where $F$ is a convex limiter of its arguments, e.g., the minmod function. 
In order to find these candidates of $U_{I+1 / 2}^{(\mathbf{m})}(\mathbf{0}),|\mathbf{m}|=m$, we take a $(m-1)$-th order partial derivative of $U_{I+1 / 2}\left(\mathbf{x}-\mathbf{x}_{I+1 / 2}\right)$, and denote it by

$$
\partial^{m-1} U_{I+1 / 2}\left(\mathbf{x}-\mathbf{x}_{I+1 / 2}\right)=L_{I+1 / 2}\left(\mathbf{x}-\mathbf{x}_{I+1 / 2}\right)+R_{I+1 / 2}\left(\mathbf{x}-\mathbf{x}_{I+1 / 2}\right),
$$

where $L_{I+1 / 2}$ is the linear part and $R_{I+1 / 2}$ contains all second and higher degree terms of $\partial^{m-1} U_{I+1 / 2}(\mathbf{x}-$ $\left.\mathbf{x}_{I+1 / 2}\right)$. Clearly, every coefficient in the first degree terms of $L_{I+1 / 2}$ is in the $\operatorname{set}\left\{U_{I+1 / 2}^{(\mathbf{m})}(\mathbf{0}):|\mathbf{m}|=m\right\}$. And for every $\mathbf{m}$ subject to $|\mathbf{m}|=m$, one can always take some $(m-1)$-th order partial derivatives of $U_{I+1 / 2}\left(\mathbf{x}-\mathbf{x}_{I+1 / 2}\right)$ so that $U_{I+1 / 2}^{(\mathbf{m})}(\mathbf{0})$ is a coefficient in the first degree terms of $L_{I+1 / 2}$. Thus, a 'candidate' for a coefficient in the first degree terms of $L_{I+1 / 2}$ is the candidate for the corresponding $U_{I+1 / 2}^{(\mathbf{m})}(\mathbf{0})$.

In order to find the candidates for all the coefficients in the first degree terms of $L_{I+1 / 2}\left(\mathbf{x}-\mathbf{x}_{I+1 / 2}\right)$, we only need to know the cell averages of $L_{I+1 / 2}\left(\mathbf{x}-\mathbf{x}_{I+1 / 2}\right)$ on $d+1$ distinct mesh cells adjacent to cell $C_{I+1 / 2}$, recalling that $d$ is the spatial dimension. Assume $C_{J_{0}}, C_{J_{1}}, \cdots, C_{J_{d}} \in\left\{C_{J}\right\}$ are these cells and $\bar{L}_{J_{0}}, \bar{L}_{J_{1}}, \cdots, \bar{L}_{J_{d}}$ are the corresponding cell averages. The set of these $d+1$ cells with the associated cell averages is called a stencil. Let a linear polynomial $\widetilde{L}_{I+1 / 2}\left(\mathbf{x}-\mathbf{x}_{I+1 / 2}\right)$ be determined by

$$
\frac{1}{\left|C_{J_{l}}\right|} \int_{C_{J_{l}}} \widetilde{L}_{I+1 / 2}\left(\mathbf{x}-\mathbf{x}_{I+1 / 2}\right) d \mathbf{x}=\bar{L}_{J_{l}}, \quad l=0,1, \cdots, d .
$$

Then the coefficients in the first degree terms of $\widetilde{L}_{I+1 / 2}\left(\mathbf{x}-\mathbf{x}_{I+1 / 2}\right)$ will be the candidates for the corresponding coefficients of $L_{I+1 / 2}\left(\mathbf{x}-\mathbf{x}_{I+1 / 2}\right)$. Therefore, a stencil located near cell $C_{I+1 / 2}$ will determine a set of candidates for all coefficients in the first degree terms of $L_{I+1 / 2}\left(\mathbf{x}-\mathbf{x}_{I+1 / 2}\right)$. The key is to determine the new approximate cell averages of $L_{I+1 / 2}\left(\mathbf{x}-\mathbf{x}_{I+1 / 2}\right)$ on the cells of $\left\{C_{J}\right\}$, which is outlined by the following algorithm.

Algorithm 1. Step 1. Suppose $r \geq 2$. For $m=r, r-1, \cdots, 2$, do the following:

(a) Take a $(m-1)$-th order partial derivative for each of $\left\{U_{J}\left(\mathbf{x}-\mathbf{x}_{J}\right)\right\}$ to obtain polynomials $\left\{\partial^{m-1} U_{J}\left(\mathbf{x}-\mathbf{x}_{J}\right)\right\}$ respectively. In particular, denote $\partial^{m-1} U_{I+1 / 2}\left(\mathbf{x}-\mathbf{x}_{I+1 / 2}\right)=L_{I+1 / 2}\left(\mathbf{x}-\mathbf{x}_{I+1 / 2}\right)+$ $R_{I+1 / 2}\left(\mathbf{x}-\mathbf{x}_{I+1 / 2}\right)$, where $L_{I+1 / 2}\left(\mathbf{x}-\mathbf{x}_{I+1 / 2}\right)$ is the linear part of $\partial^{m-1} U_{I+1 / 2}\left(\mathbf{x}-\mathbf{x}_{I+1 / 2}\right)$ and $R_{I+1 / 2}\left(\mathbf{x}-\mathbf{x}_{I+1 / 2}\right)$ is the remainder.

(b) Calculate the cell averages of $\left\{\partial^{m-1} U_{J}\left(\mathbf{x}-\mathbf{x}_{J}\right)\right\}$ on cells $\left\{C_{J}\right\}$ to obtain $\left\{\overline{\partial^{m-1} U_{J}}\right\}$ respectively.

(c) Let $\widetilde{R}_{I+1 / 2}\left(\mathbf{x}-\mathbf{x}_{I+1 / 2}\right)$ be the $R_{I+1 / 2}\left(\mathbf{x}-\mathbf{x}_{I+1 / 2}\right)$ with its coefficients replaced by the corresponding new coefficients ${ }^{3}$. Calculate the cell averages of $\widetilde{R}_{I+1 / 2}\left(\mathbf{x}-\mathbf{x}_{I+1 / 2}\right)$ on cells $\left\{C_{J}\right\}$ to obtain $\left\{\bar{R}_{J}\right\}$ respectively.

(d) Let $\bar{L}_{J}=\overline{\partial^{m-1} U_{J}}-\bar{R}_{J}$ for all $J$.

(e) Form stencils out of the new approximate cell averages $\left\{\bar{L}_{J}\right\}$ by using a non-oscillatory finite volume MUSCL or second order ENO strategy. Each stencil will determine a set of candidates for the coefficients in the first degree terms of $L_{I+1 / 2}\left(\mathbf{x}-\mathbf{x}_{I+1 / 2}\right)$, which are also candidates for the corresponding $U_{I+1 / 2}^{(\mathbf{m})}(\mathbf{0}) ' s,|\mathbf{m}|=m$.

(f) Repeat from (a) to (e) until all possible combinations of the $(m-1)$-th order partial derivatives are taken. Then the candidates for all coefficients in the $m$-th degree terms of $U_{I+1 / 2}\left(\mathbf{x}-\mathbf{x}_{I+1 / 2}\right)$

\footnotetext{
${ }^{3}$ At this stage, we have already found new values for all coefficients in the terms of $U_{I+1 / 2}\left(\mathbf{x}-\mathbf{x}_{I+1 / 2}\right)$ of degree above $m$. These coefficients remain in $R_{I+1 / 2}\left(\mathbf{x}-\mathbf{x}_{I+1 / 2}\right)$ (after taking a $(m-1)$-th order partial of $U_{I+1 / 2}\left(\mathbf{x}-\mathbf{x}_{I+1 / 2}\right)$ ). When they are replaced by their corresponding new values, $R_{I+1 / 2}\left(\mathbf{x}-\mathbf{x}_{I+1 / 2}\right)$ becomes $\widetilde{R}_{I+1 / 2}\left(\mathbf{x}-\mathbf{x}_{I+1 / 2}\right)$. See Step $2(2)$ in Sec. 4.1 as an example.
} 
have been computed. For each of these coefficients, say $\frac{1}{\mathbf{m} !} U_{I+1 / 2}^{(\mathbf{m})}(\mathbf{0}),|\mathbf{m}|=m$, let the new coefficient $\widetilde{U}_{I+1 / 2}^{(\mathbf{m})}(\mathbf{0})=F\left(\right.$ candidates of $\left.U_{I+1 / 2}^{(\mathbf{m})}(\mathbf{0})\right)$.

Step 2. In order to find the new coefficients in the zero-th and first degree terms of $U_{I+1 / 2}(\mathbf{x}-$ $\left.\mathbf{x}_{I+1 / 2}\right)$, we perform the procedure of Step 1 (a)-(f) with $m=1$, and make sure that the new approximate cell average $\bar{L}_{I+1 / 2}$ is in each of the stencils, which ensures that the cell average of $U_{I+1 / 2}\left(\mathbf{x}-\mathbf{x}_{I+1 / 2}\right)$ on cell $C_{I+1 / 2}$ is not changed with the new coefficients. The new coefficient in the zero-th degree term of $U_{I+1 / 2}\left(\mathbf{x}-\mathbf{x}_{I+1 / 2}\right)$ is $\bar{L}_{I+1 / 2}$, which ensures that the cell average of $U_{I+1 / 2}\left(\mathbf{x}-\mathbf{x}_{I+1 / 2}\right)$ in cell $C_{I+1 / 2}$ is invariant with the new coefficients. At this stage all new coefficients of $U_{I+1 / 2}\left(\mathbf{x}-\mathbf{x}_{I+1 / 2}\right)$ have been found.

Remarks. 1. The coefficients of the polynomials can be updated after Algorithm 1 has been applied to all mesh cells, or at the $m$-th stage when all new coefficients for those in the $m$-th degree terms of all polynomials have been computed (in this case, $\left\{\overline{\partial^{0} U_{J}}\right\}$ used in Step 2 should be the cell averages of the original polynomials to ensure that they are invariant). The latter case is supposed to be more diffusive. In numerical experiments we find their results are about the same. All numerical results presented in this paper are performed with the former implementation.

2. One motivation for us to develop this hierarchical reconstruction is that the limiting for the DG scheme on non-staggered meshes is different from that for scheme (3.4). For the usual DG scheme the time evolution of the cell averages is completely determined by the fluxes, however in (3.4), cell interior values are also involved. We find in numerical experiments that the moment limiter [7] does not work as robustly for scheme (3.4) as it does for DG scheme on non-staggered meshes. The proposed hierarchical reconstruction process is quite general and could be useful for conventional DG or even finite volume schemes. These will be explored in the future.

3. Scheme (3.4) with Algorithm 1 and with piece-wise linear elements is identical to the second order central scheme on overlapping cells [37].

4. It is more efficient to apply the hierarchical reconstruction process only at places where it is needed by using non-smoothness detectors (see e.g. [42, 9]). This will be explored in the future.

The most important point is that, even though the linear reconstruction used in Algorithm 1 is only second order accurate, the approximation order of accuracy of a polynomial in a cell is unaffected by the algorithm and we have the following theorem.

Condition 1. Let $\left\{\mathbf{x}_{J_{0}}, \mathbf{x}_{J_{1}}, \cdots, \mathbf{x}_{J_{d}}\right\}$ be the $d+1$ cell centroids of a stencil. Then there is a point among them, say $\mathbf{x}_{J_{0}}$, such that the matrix $A=\frac{1}{\Delta x}\left[\mathbf{x}_{J_{1}}-\mathbf{x}_{J_{0}}, \mathbf{x}_{J_{2}}-\mathbf{x}_{J_{0}}, \cdots, \mathbf{x}_{J_{d}}-\mathbf{x}_{J_{0}}\right]$ is non singular. Further, there is a constant $\alpha>0$ independent of $\Delta x$ such that $\left\|A^{-1}\right\| \leq \alpha$.

In $2 \mathrm{D}$, this condition means that $\mathbf{x}_{J_{0}}, \mathbf{x}_{J_{1}}, \mathbf{x}_{J_{2}}$ are not along a straight line. Further, the angle between the line passing $\mathbf{x}_{J_{0}}, \mathbf{x}_{J_{1}}$ and the line passing $\mathbf{x}_{J_{0}}, \mathbf{x}_{J_{2}}$ has a positive lower bound independent of $\Delta x$. This condition is satisfied for stencils such as $\left\{C_{3}, C_{1}, C_{2}\right\},\left\{C_{3}, C_{2}, C_{5}\right\},\left\{C_{3}, C_{5}, C_{4}\right\}$ and $\left\{C_{3}, C_{4}, C_{1}\right\}$ in figure 3 right; and is not satisfied for $\left\{C_{1}, C_{3}, C_{5}\right\}$.

Theorem 2. Suppose $\left\{U_{J}\left(\mathbf{x}-\mathbf{x}_{J}\right)\right\}$ in Algorithm 1 approximate a $C^{r+1}$ function $u(\mathbf{x})$ with point-wise error $\mathcal{O}\left((\Delta x)^{r+1}\right)$ within their respective cell $\left\{C_{J}\right\}$, and all cells in $\left\{C_{J}\right\}$ are contained in a circle centered at $\mathbf{x}_{I+1 / 2}$ with radius $\mathcal{O}(\Delta x)$. Let the $d+1$ cell centroids in every stencil used in Algorithm 1 satisfy Condition 1. Then after the application of Algorithm 1, the polynomial $\widetilde{U}_{I+1 / 2}\left(\mathbf{x}-\mathbf{x}_{I+1 / 2}\right)$, i.e. $U_{I+1 / 2}\left(\mathbf{x}-\mathbf{x}_{I+1 / 2}\right)$ with its coefficients replaced by the corresponding new values also approximates the function $u(\mathbf{x})$ with point-wise error $\mathcal{O}\left((\Delta x)^{r+1}\right)$ within cell $C_{I+1 / 2}$. The cell average of $\widetilde{U}_{I+1 / 2}(\mathbf{x}-$ $\left.\mathbf{x}_{I+1 / 2}\right)$ on cell $C_{I+1 / 2}$ is the same as that of $U_{I+1 / 2}\left(\mathbf{x}-\mathbf{x}_{I+1 / 2}\right)$. 
Proof. From the assumption we know that the coefficients in the $m$-th degree terms of $U_{I+1 / 2}(\mathbf{x}-$ $\left.\mathbf{x}_{I+1 / 2}\right), 0 \leq m \leq r$, are the $(r-m+1)$-th order approximation to the corresponding coefficients of the Taylor expansion of $u(\mathbf{x})$ at $\mathbf{x}_{I+1 / 2}$.

Assume that when starting to compute new values for the coefficients of the $m$-th degree terms of $U_{I+1 / 2}\left(\mathbf{x}-\mathbf{x}_{I+1 / 2}\right), 1 \leq m \leq r$, all the computed new values (if there is any) for the coefficients of the $l$-th degree terms $\left(m<l \leq r\right.$, if they exist) of $U_{I+1 / 2}\left(\mathbf{x}-\mathbf{x}_{I+1 / 2}\right)$ are their $(r-l+1)$-th order approximations. In fact, when $m=r$, there is no new coefficients which have been computed at Step 1 (a). However, the following argument will show that the new coefficients computed at Step 1 (f) for coefficients of the $r$-th degree terms of $U_{I+1 / 2}\left(\mathbf{x}-\mathbf{x}_{I+1 / 2}\right)$ are their first order approximations.

Let $L_{I+1 / 2}\left(\mathbf{x}-\mathbf{x}_{I+1 / 2}\right)=c_{0}+\mathbf{c}_{1} \cdot\left(\mathbf{x}-\mathbf{x}_{I+1 / 2}\right)$ in Step 1 (a) and let $\widehat{L}(\mathbf{x})=\widehat{c}_{0}+\widehat{\mathbf{c}}_{1} \cdot\left(\mathbf{x}-\mathbf{x}_{I+1 / 2}\right)$ be the corresponding linear part in the Taylor expansion of the same ( as for $\left.U_{J}\right)(m-1)$-th partial derivative of $u(x)$ at $\mathbf{x}_{I+1 / 2}$. Therefore $c_{0}$ and $\mathbf{c}_{1}$ approximate $\widehat{c}_{0}$ and $\widehat{\mathbf{c}}_{1}$ to the order of $\mathcal{O}\left((\Delta x)^{r-m+2}\right)$ and $\mathcal{O}\left((\Delta x)^{r-m+1}\right)$ respectively. Also from the above assumptions it is easy to see that $\bar{L}_{J}=\overline{\partial^{m-1} U_{J}}-\bar{R}_{J}$ in Step 1 (d) approximates the cell average of $\widehat{L}(\mathbf{x})$ on cell $C_{J}$ to the order of $O\left(\Delta x^{r-m+2}\right)$, for all cells $C_{J}$ adjacent to cell $C_{I+1 / 2}$.

Reconstructing $\widetilde{L}_{I+1 / 2}\left(\mathbf{x}-\mathbf{x}_{I+1 / 2}\right)=\widetilde{c}_{0}+\widetilde{\mathbf{c}}_{1} \cdot\left(\mathbf{x}-\mathbf{x}_{I+1 / 2}\right)$ from a stencil $C_{J_{0}}, C_{J_{1}}, \cdots, C_{J_{d}} \in\left\{C_{J}\right\}$ is to find $\widetilde{c}_{0}$ and $\widetilde{\mathbf{c}}_{1}$ satisfying the following equations (see (4.2)),

$$
\begin{aligned}
\frac{1}{\left|C_{J_{l}}\right|} \int_{C_{J_{l}}}\left(\widetilde{c}_{0}+\widetilde{\mathbf{c}}_{1} \cdot\left(\mathbf{x}-\mathbf{x}_{I+1 / 2}\right)\right) d \mathbf{x} & =\widetilde{c}_{0}+\widetilde{\mathbf{c}}_{1} \cdot\left(\mathbf{x}_{J_{l}}-\mathbf{x}_{I+1 / 2}\right) \\
& =\bar{L}_{J_{l}}=\widehat{c}_{0}+\widehat{\mathbf{c}}_{1} \cdot\left(\mathbf{x}_{J_{l}}-\mathbf{x}_{I+1 / 2}\right)+\mathcal{O}\left((\Delta x)^{r-m+2}\right)
\end{aligned}
$$

where $\mathbf{x}_{J_{l}}$ is the cell centroid of cell $C_{J_{l}}, l=0, \cdots, d$. The solutions are candidates for $c_{0}$ and $\mathbf{c}_{1}$ respectively. Subtracting the first equation $(l=0)$ from the rest of the equations in (4.3) we can obtain

$$
A^{T}\left(\widetilde{\mathbf{c}}_{1}-\widehat{\mathbf{c}}_{1}\right)=\mathcal{O}\left((\Delta x)^{r-m+1}\right),
$$

where $A=\frac{1}{\Delta x}\left[\mathbf{x}_{J_{1}}-\mathbf{x}_{J_{0}}, \mathbf{x}_{J_{2}}-\mathbf{x}_{J_{0}}, \cdots, \mathbf{x}_{J_{d}}-\mathbf{x}_{J_{0}}\right]$. From Condition $1,\left\|A^{-1}\right\|$ is bounded independent of $\Delta x$. We conclude that the candidate

$$
\widetilde{\mathbf{c}}_{1}=\widehat{\mathbf{c}}_{1}+\mathcal{O}\left((\Delta x)^{r-m+1}\right) .
$$

Also since $\left\|\mathbf{x}_{J_{l}}-\mathbf{x}_{I+1 / 2}\right\|=\mathcal{O}(\Delta x), l=0,1, \cdots, d$, by substituting the estimate of the candidate $\widetilde{\mathbf{c}}_{1}$ back into one of the equations of (4.3) we obtain that the candidate

$$
\widetilde{c}_{0}=\widehat{c}_{0}+\mathcal{O}\left((\Delta x)^{r-m+2}\right) \text {. }
$$

Since the function $F$ used in Step 1 (f) is a convex combination of its arguments, it does not change the approximation order of its arguments. Therefore estimate (4.4) implies that the new values for coefficients of the $m$-th degree terms of $U_{I+1 / 2}\left(\mathbf{x}-\mathbf{x}_{I+1 / 2}\right)$ are their $(r-m+1)$-th order approximations. Estimate (4.4) moves the induction till $m=1$ and estimate (4.5) implies that in Step 2, the new value for the coefficient of the zero-th degree term of $U_{I+1 / 2}\left(\mathbf{x}-\mathbf{x}_{I+1 / 2}\right)$ is its $O\left(\Delta x^{r+1}\right)$ approximation. Step 2 clearly ensures that the cell average of $U_{I+1 / 2}\left(\mathbf{x}-\mathbf{x}_{I+1 / 2}\right)$ on cell $C_{I+1 / 2}$ is unchanged with the new coefficients. The proof is now complete.

4.3. Implementation for Piece-wise Quadratic Finite Element Space in 2D. Suppose on cell $C_{j}$ (see figure 3 right), a quadratic polynomial is given as

$$
\begin{aligned}
U_{j}\left(x-x_{j}, y-y_{j}\right)= & U_{j}(0,0)+\partial_{x} U_{j}(0,0)\left(x-x_{j}\right)+\partial_{y} U_{j}(0,0)\left(y-y_{j}\right)+ \\
& \frac{1}{2} \partial_{x x} U_{j}(0,0)\left(x-x_{j}\right)^{2}+\partial_{x y} U_{j}(0,0)\left(x-x_{j}\right)\left(y-y_{j}\right)+\frac{1}{2} \partial_{y y} U_{j}(0,0)\left(y-y_{j}\right)^{2},
\end{aligned}
$$

where $\left(x_{j}, y_{j}\right)$ is the cell centroid of cell $C_{j}, j=1,2, \cdots, 5$. 


\begin{tabular}{|c|c|c|c|c|c|}
\hline$\Delta x$ & $1 / 10$ & $1 / 20$ & $1 / 40$ & $1 / 80$ & $1 / 160$ \\
\hline$L_{1}$ error & $4.24 \mathrm{E}-4$ & $5.33 \mathrm{E}-5$ & $6.71 \mathrm{E}-6$ & $8.44 \mathrm{E}-7$ & $1.07 \mathrm{E}-7$ \\
\hline order & - & 2.99 & 2.99 & 2.99 & 2.98 \\
\hline$L_{\infty}$ error & $5.13 \mathrm{E}-4$ & $6.20 \mathrm{E}-5$ & $7.38 \mathrm{E}-6$ & $1.29 \mathrm{E}-6$ & $2.66 \mathrm{E}-7$ \\
\hline order & - & 3.05 & 3.07 & 2.52 & 2.28 \\
\hline
\end{tabular}

TABLE 8. $P^{2}$ version of the central DG scheme (3.4) with the hierarchical reconstruction Algorithm 1 for the Burgers' equation. MUSCL is used in Algorithm 1.

\begin{tabular}{|c|c|c|c|c|c|}
\hline$\Delta x$ & $1 / 10$ & $1 / 20$ & $1 / 40$ & $1 / 80$ & $1 / 160$ \\
\hline$L_{1}$ error & $4.51 \mathrm{E}-4$ & $5.36 \mathrm{E}-5$ & $6.85 \mathrm{E}-6$ & $8.54 \mathrm{E}-7$ & $1.08 \mathrm{E}-7$ \\
\hline order & - & 3.07 & 2.97 & 3.00 & 2.98 \\
\hline$L_{\infty}$ error & $5.24 \mathrm{E}-4$ & $6.17 \mathrm{E}-5$ & $1.03 \mathrm{E}-5$ & $1.81 \mathrm{E}-6$ & $3.27 \mathrm{E}-7$ \\
\hline order & - & 3.09 & 2.58 & 2.51 & 2.47 \\
\hline
\end{tabular}

TABLE 9. $P^{2}$ version of the central DG scheme (3.4) with the hierarchical reconstruction Algorithm 1 for the Burgers' equation. Second order ENO is used in Algorithm 1.

\begin{tabular}{|c|c|c|c|c|c|}
\hline$\Delta x$ & $1 / 4$ & $1 / 8$ & $1 / 16$ & $1 / 32$ & $1 / 64$ \\
\hline$L_{1}$ error & $8.00 \mathrm{E}-2$ & $1.24 \mathrm{E}-2$ & $1.58 \mathrm{E}-3$ & $1.92 \mathrm{E}-4$ & $2.40 \mathrm{E}-5$ \\
\hline order & - & 2.69 & 2.97 & 3.04 & 3.00 \\
\hline$L_{\infty}$ error & $4.90 \mathrm{E}-2$ & $9.85 \mathrm{E}-3$ & $1.68 \mathrm{E}-3$ & $2.01 \mathrm{E}-4$ & $2.68 \mathrm{E}-5$ \\
\hline order & - & 2.31 & 2.55 & 3.06 & 2.91 \\
\hline
\end{tabular}

TABLE 10. $P^{2}$ version of the central DG scheme (3.4) with the hierarchical reconstruction Algorithm 1 for the 2D Burgers' equation. Second order ENO is used in Algorithm 1.

According to Step 1 of Algorithm 1, take the first partial derivative with respect to $x$ for them to obtain $L_{j}\left(x-x_{j}, y-y_{j}\right)=\partial_{x} U_{j}(0,0)+\partial_{x x} U_{j}(0,0)\left(x-x_{j}\right)+\partial_{x y} U_{j}(0,0)\left(y-y_{j}\right), j=1,2, \cdots, 5$. Calculate the cell average of $L_{j}\left(x-x_{j}, y-y_{j}\right)$ on cell $C_{j}$ to obtain $\bar{L}_{j}=\partial_{x} U_{j}(0,0), j=1,2, \cdots, 5$ (note that $R_{3}\left(x-x_{3}, y-y_{3}\right) \equiv 0$ ). With the five new approximate cell averages $\left\{\bar{L}_{j}: j=1,2, \cdots, 5\right\}$, one can apply a MUSCL or a second order ENO procedure to reconstruct a non-oscillatory linear polynomial

$$
\widetilde{L}_{3}\left(x-x_{3}, y-y_{3}\right)=\partial_{x} \widetilde{U}_{3}(0,0)+\partial_{x x} \widetilde{U}_{3}(0,0)\left(x-x_{3}\right)+\partial_{x y} \widetilde{U}_{3}(0,0)\left(y-y_{3}\right)
$$

in cell $C_{3}$. For example, one can form four stencils $\left\{C_{3}, C_{1}, C_{2}\right\},\left\{C_{3}, C_{2}, C_{5}\right\},\left\{C_{3}, C_{5}, C_{4}\right\}$ and $\left\{C_{3}, C_{4}, C_{1}\right\}$. For the first stencil, solve the following equations for $\partial_{x x} \widetilde{U}_{3}(0,0)$ and $\partial_{x y} \widetilde{U}_{3}(0,0)$

$$
\begin{aligned}
\frac{1}{\left|C_{j}\right|} \int_{C_{j}} \widetilde{L}_{3}\left(x-x_{3}, y-y_{3}\right) d x d y & =\bar{L}_{3}+\partial_{x x} \widetilde{U}_{3}(0,0)\left(x_{j}-x_{3}\right)+\partial_{x y} \widetilde{U}_{3}(0,0)\left(y_{j}-y_{3}\right) \\
& =\bar{L}_{j}
\end{aligned}
$$

$j=1,2$, similarly for other stencils. We obtain two sets of candidates for $\partial_{x x} U_{3}(0,0)$ and $\partial_{x y} U_{3}(0,0)$ respectively. By taking the first partial derivative with respect to $y$ for $U_{j}\left(x-x_{j}, y-y_{j}\right), j=1,2, \cdots, 5$, we similarly obtain a set of candidates for $\partial_{y y} U_{3}(0,0)$ and enlarge the set of candidates for $\partial_{x y} U_{3}(0,0)$. Putting all candidates for $\partial_{x x} U_{3}(0,0)$ into the arguments of the minmod (or $\operatorname{minmod} 2$ ) function, we 
obtain the new coefficient $\partial_{x x} \widetilde{U}_{3}(0,0)$ for $\partial_{x x} U_{3}(0,0)$. Applying the same procedure to obtain new coefficients $\partial_{x y} \widetilde{U}_{3}(0,0)$ and $\partial_{y y} \widetilde{U}_{3}(0,0)$.

According to Step 2 of Algorithm 1, we compute the cell average of $U_{j}\left(x-x_{j}, y-y_{j}\right)$ on cell $C_{j}$ to obtain $\overline{U_{j}}, j=1,2, \cdots, 5$; and compute cell averages of the polynomial

$$
\widetilde{R}_{3}\left(x-x_{3}, y-y_{3}\right)=\frac{1}{2} \partial_{x x} \widetilde{U}_{3}(0,0)\left(x-x_{3}\right)^{2}+\partial_{x y} \widetilde{U}_{3}(0,0)\left(x-x_{3}\right)\left(y-y_{3}\right)+\frac{1}{2} \partial_{y y} \widetilde{U}_{3}(0,0)\left(y-y_{3}\right)^{2}
$$

on cell $C_{1}, C_{2}, \cdots, C_{5}$ to obtain $\bar{R}_{1}, \bar{R}_{2}, \cdots, \bar{R}_{5}$ respectively. Redefine $\bar{L}_{j}=\overline{U_{j}}-\bar{R}_{j}, j=1,2, \cdots, 5$. The same MUSCL or second order ENO procedure as described previously can be applied to the five cell averages $\left\{\bar{L}_{j}: j=1,2, \cdots, 5\right\}$ to obtain the new coefficients $\partial_{x} \widetilde{U}_{3}(0,0)$ and $\partial_{y} \widetilde{U}_{3}(0,0)$. Finally let the new coefficient $\widetilde{U}_{3}(0,0)=\bar{L}_{3}$.

The convergence test results with Algorithm 1 for Example 3 can be found in Table 10. We again observe that the order of accuracy is maintained, although (as expected for any limiter) the magnitude of the error is increased for the same mesh (see Table 6 for a comparison).

\section{Additional Numerical Examples}

Scheme (3.4) with the piece-wise $r$-th degree polynomial space is referred to as CO-DG- $(r+1)$, where "C" stands for "Central" and "O" stands for "Overlapping cells". When the hierarchical reconstruction Algorithm 1 is applied, it is referred to as CO-DG-hr1- $(r+1)$. To specify whether a linear MUSCL (with the minmod limiter) or ENO (with the $\operatorname{minmod}_{2}$ limiter) reconstruction is used in Algorithm 1, we refer it as CO-DG-hr1m- $(r+1)$ or CO-DG-hr1e- $(r+1)$ respectively.

The corresponding (up to third order) TVD Runge-Kutta time discretization methods [45] are applied to the above schemes. Only the solution in one class of the overlapping cells is shown in the graphs throughout this section. For systems of equations, the component-wise extensions of the scalar schemes (without characteristic decomposition) have been used in all the computations.

Example 5. We compute the Euler equation with Lax's initial data. $u_{t}+f(u)_{x}=0$ with $u=$ $(\rho, \rho v, E)^{T}, f(u)=\left(\rho v, \rho v^{2}+p, v(E+p)\right)^{T}, p=(\gamma-1)\left(E-\frac{1}{2} \rho v^{2}\right), \gamma=1.4$. Initially, the density $\rho$, momentum $\rho v$ and total energy $E$ are $0.445,0.311$ and 8.928 in $(0,0.5)$; and are $0.5,0$ and 1.4275 in $(0.5,1)$. The computed results by CO-DG-hr1e-3 and CO-DG-hr1m-3 are shown at $T=0.16$ in figure 4 , with $\Delta x=1 / 200, \Delta \tau^{n}$ chosen with a CFL factor $0.4, \Delta t^{n}=0.5 \Delta \tau^{n}$. The solid line reference solutions are analytic solutions to the Riemann problem. We observe that the resolution is quite good with very small over/undershoots. The only concern is that the contact discontinuity is much more smeared than that of the regular third order DG scheme with a TVB limiter (Figure 20 in [11]). We hope to improve this performance by reducing the usage of the reconstruction limiter through a troubled-cell indicator in future work.

Example 6. The Woodward and Colella blast wave problem [50] for the Euler equation computed by CO-DG-hr1e-3. Initially, the density, momentum, total energy are 1, 0, 2500 in $(0,0.1) ; 1,0,0.025$ in $(0.1,0.9)$; and $1,0,250$ in $(0.9,1)$. The density, velocity and pressure profiles are plotted in figure 5 for $T=0.01$ and $T=0.038$. The solid line reference solutions are computed by a $3 r d$ order central scheme on overlapping cells [37] on a much refined mesh $(\Delta x=1 / 2000) . \Delta \tau^{n}$ is chosen with a CFL factor $0.4, \Delta t^{n}=\frac{1}{2} \Delta \tau^{n}$. We observe stable results with good resolution for this very demanding problem in terms of numerical stability.

Example 7. Shu-Osher problem [46]. It is the Euler equation with an initial data

$$
\begin{aligned}
& (\rho, v, p)=(3.857143,2.629369,10.333333), \quad \text { for } x<-4 \\
& (\rho, v, p)=(1+0.2 \sin (5 x), 0,1), \quad \text { for } x \geq-4 .
\end{aligned}
$$



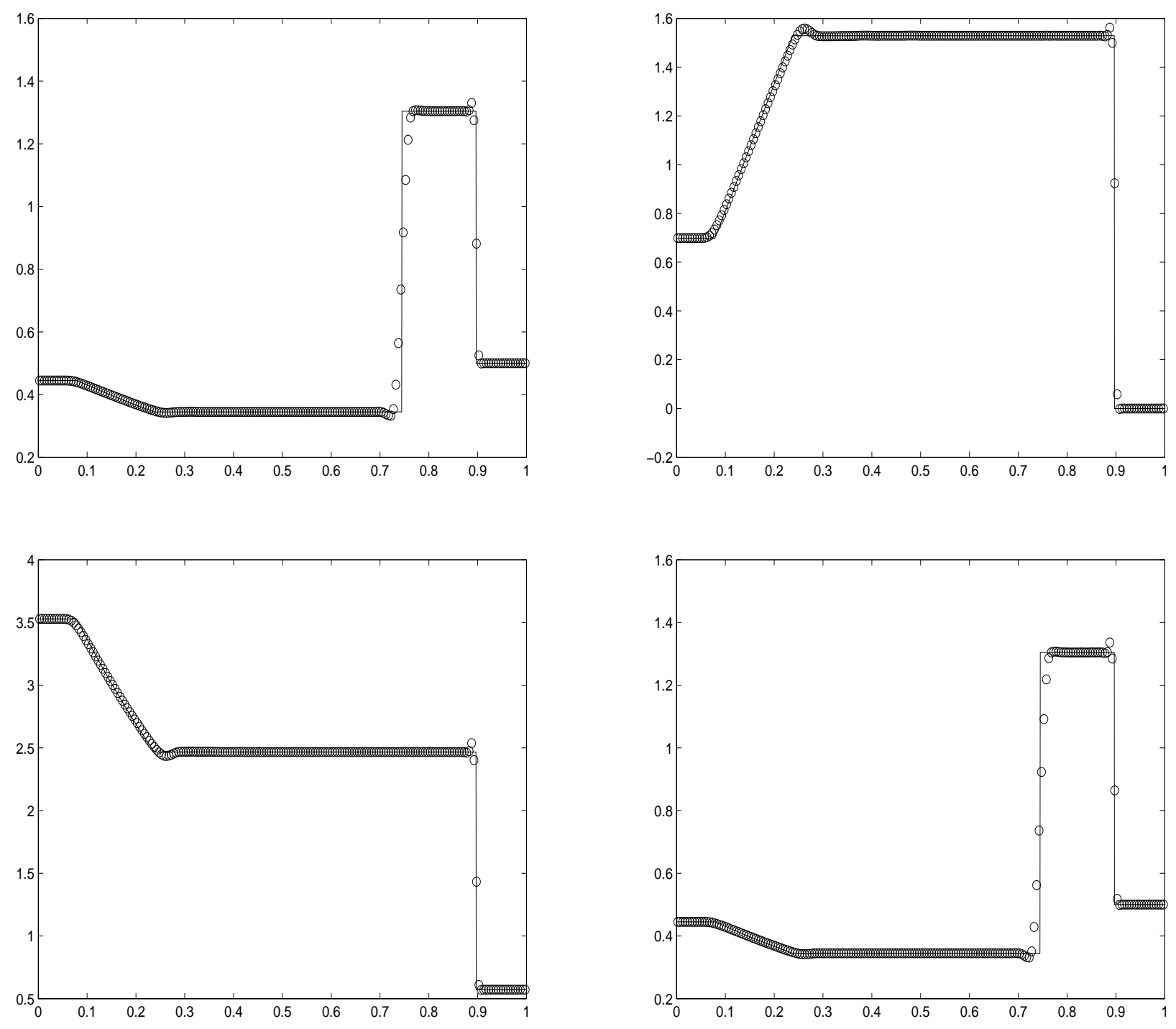

Figure 4: Lax's Problem. $\Delta x=1 / 200$. From left to right, top to bottom, (1) density (CO-DG-hr1e-3); (2) velocity (CO-DG-hr1e-3); (3) pressure (CO-DG-hr1e-3); (4) density (CO-DG-hr1m-3).

The density profiles are plotted at $T=1.8$, with $\Delta x=1 / 40$, see figure $6 . \Delta \tau^{n}$ is chosen with a CFL factor $0.5, \Delta t^{n}=0.5 \Delta \tau^{n}$. The solid line is the numerical solution on a fine mesh $(\Delta x=1 / 200)$ computed by a central scheme on overlapping cells [37]. We observe very good resolution for this example. In order to see the resolution of the 2D non-oscillatory hierarchical reconstruction algorithm, we put the Shu-Osher problem to a $2 \mathrm{D}$ domain $[-5,5] \times[0,0.25]$ and solve the $2 \mathrm{D}$ Euler equation. Initially the density variation is only along the $x$ direction. The density profiles at $T=1.8$ are plotted in figure 7. 

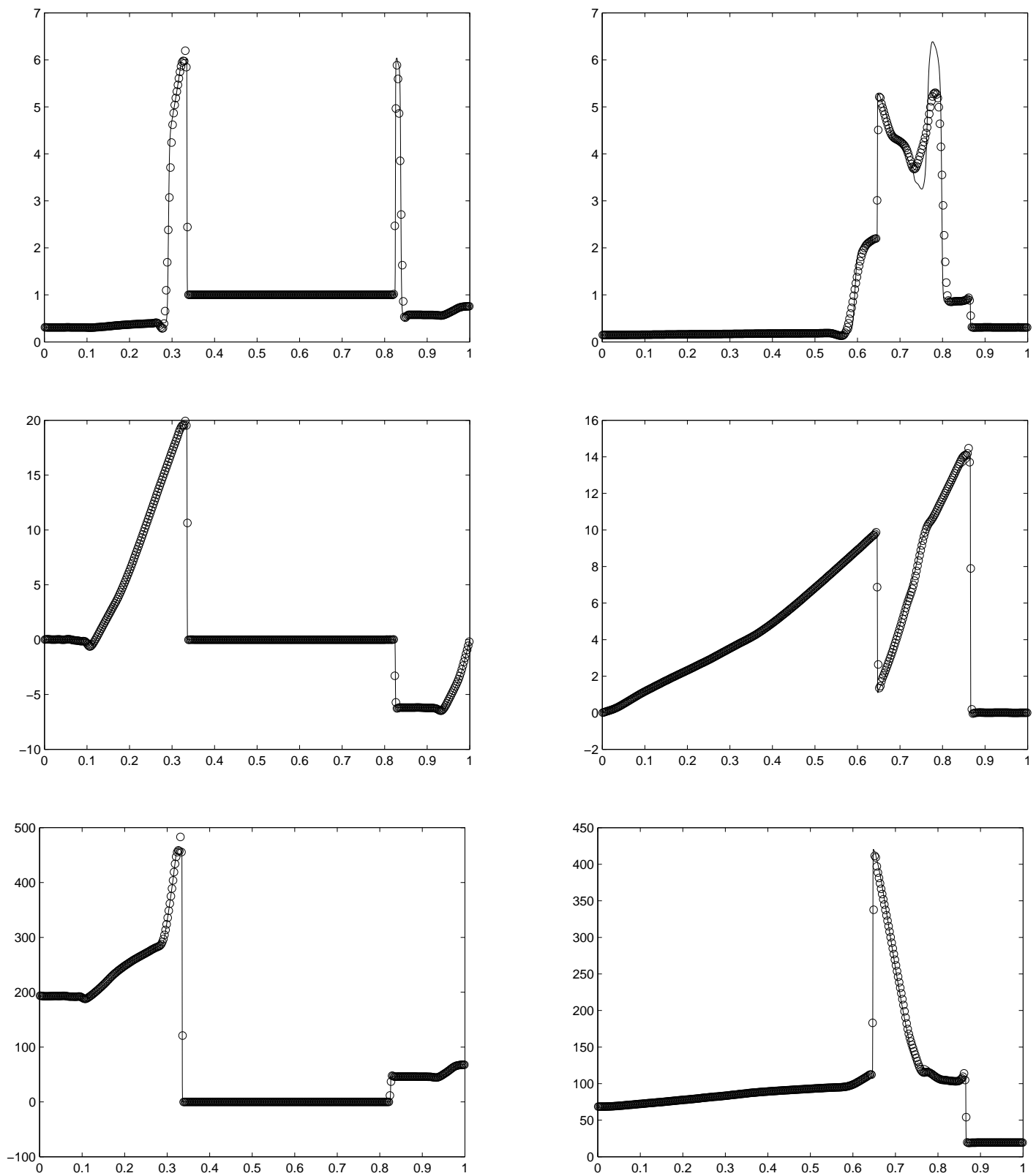

Figure 5: Woodward and Colella blast wave problem computed by CO-DG-hr1e-3. $\Delta x=1 / 400$. Top: density; middle: velocity; bottom: pressure. Left: $T=0.01$; right: $T=0.038$.

Example 8. 2D Riemann problem [29] for the Euler equation computed by CO-DG-hr1e-3. The 2D Euler equation can be written as

$$
\begin{aligned}
& \mathbf{u}_{t}+\mathbf{f}(\mathbf{u})_{x}+\mathbf{g}(\mathbf{u})_{y}=0, \mathbf{u}=(\rho, \rho u, \rho v, E)^{T}, p=(\gamma-1)\left(E-\frac{1}{2} \rho\left(u^{2}+v^{2}\right)\right) \\
& \mathbf{f}(\mathbf{u})=\left(\rho u, \rho u^{2}+p, \rho u v, u(E+p)\right)^{T}, \mathbf{g}(\mathbf{u})=\left(\rho v, \rho u v, \rho v^{2}+p, v(E+p)\right)^{T},
\end{aligned}
$$

where $\gamma=1.4$. The computational domain is $[0,1] \times[0,1]$. The initial states are constants within each of the 4 quadrants. Counter-clock-wisely from the upper right quadrant, they are labeled as $\left(\rho_{i}, u_{i}, v_{i}, p_{i}\right), i=1,2,3,4$. Initially, $\rho_{1}=1.1, u_{1}=0, v_{1}=0, p_{1}=1.1 ; \rho_{2}=0.5065, u_{2}=0.8939$, 

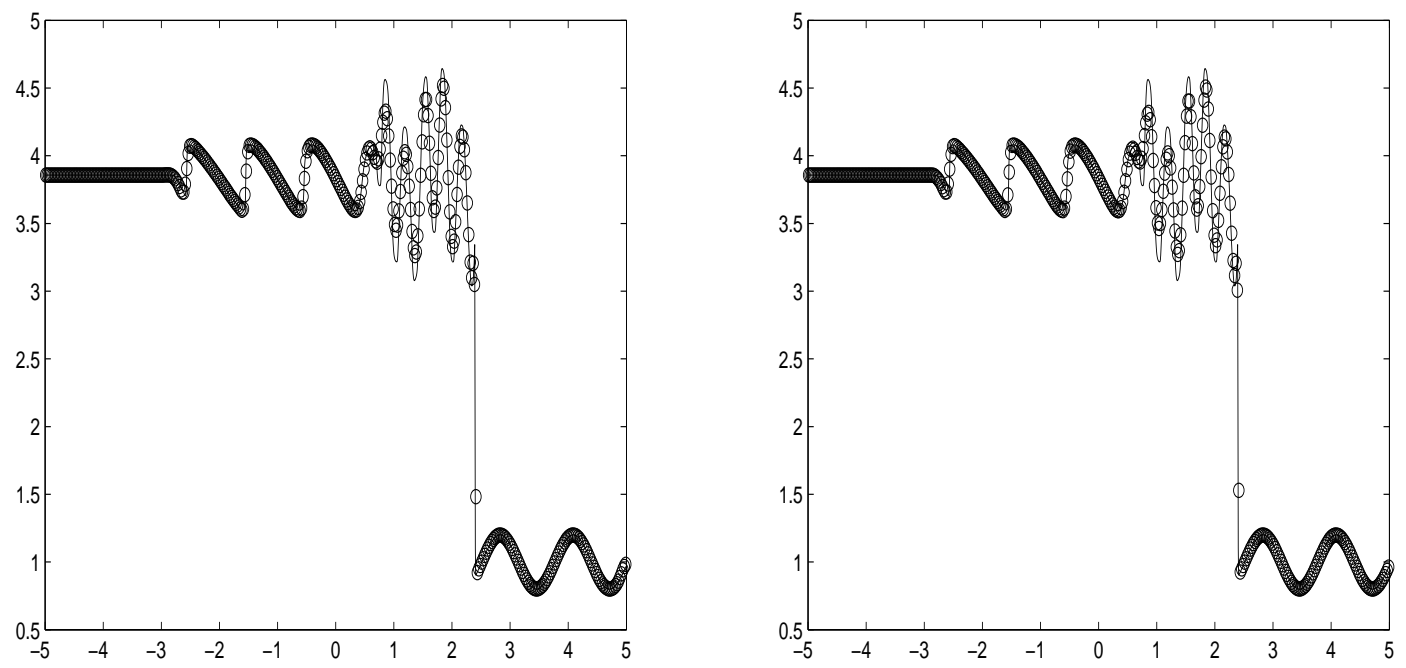

Figure 6: Shu-Osher Problem, $\Delta x=1 / 40$. Left: CO-DG-dr1m-3. Right: CO-DG-hr1e-3.
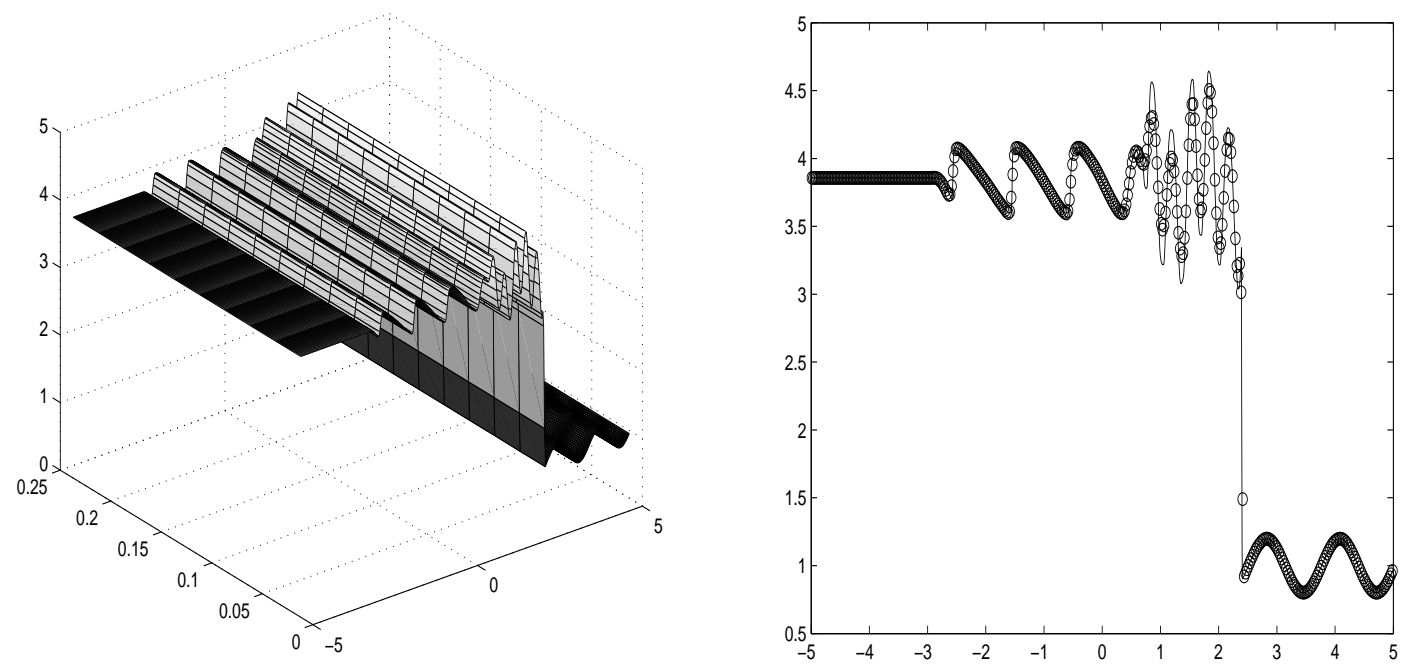

Figure 7: Shu-Osher Problem in 2D, $\Delta x=\Delta y=1 / 40$. CO-DG-hr1e-3. Left: density in the $x y$ plane. Right: density along the line $y=0.25 / 3$.

$v_{2}=0, p_{2}=0.35 ; \rho_{3}=1.1, u_{3}=0.8939, v_{3}=0.8939, p_{3}=1.1 ;$ and $\rho_{4}=0.5065, u_{4}=0, v_{4}=0.8939$, $p_{4}=0.35$. The density and pressure profiles are plotted at $T=0.25$ in figure 8 , with 30 equally spaced contours. The numerical resolution is quite good for this problem. The density profile along $y=1 / 3$ is plotted in figure 9. There is no oscillation near the discontinuities.

Example 9. Double Mach reflection [50] computed by CO-DG-hr1e-3. A planar Mach 10 shock is incident on an oblique wedge at a $\pi / 3$ angle. The air in front of the shock has density 1.4 , pressure 1 and velocity 0 . The boundary condition is described in [50]. The density and pressure profiles are plotted at $T=0.2$ in figure 10 , with 30 equally spaced contours. $\Delta x=\Delta y=1 / 120, \Delta \tau^{n}$ chosen with 

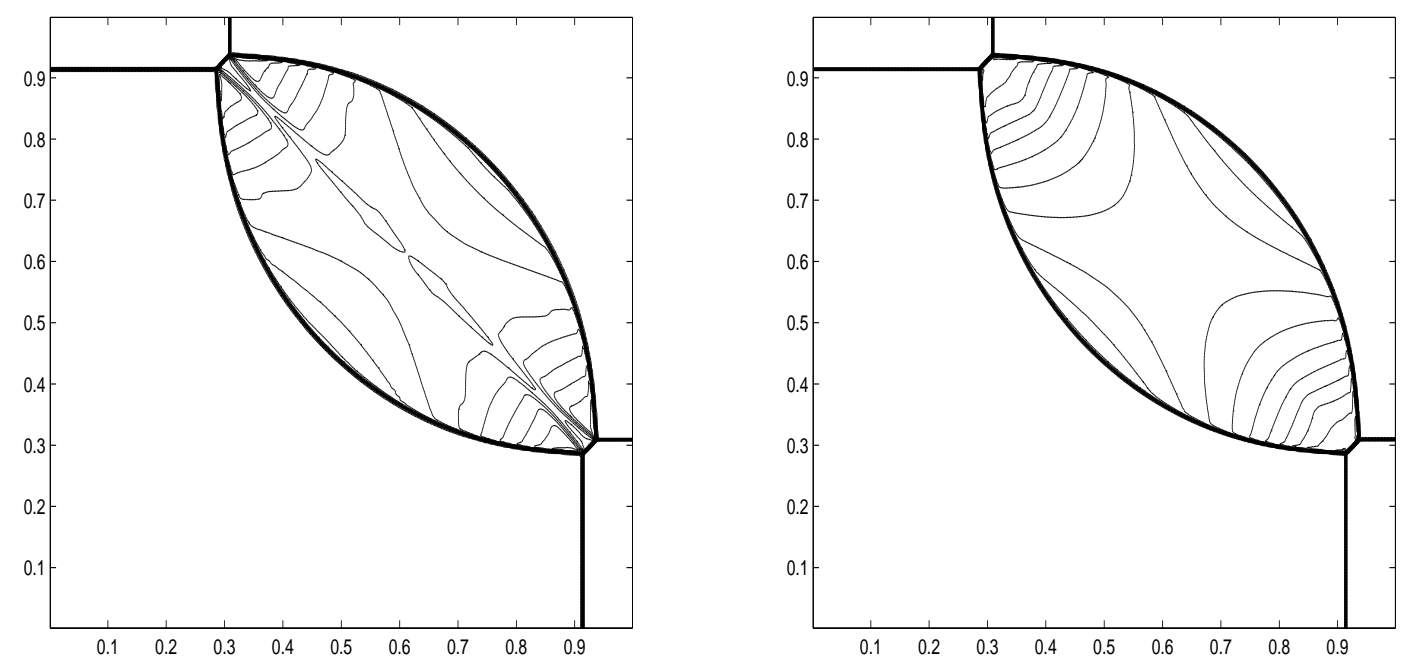

Figure 8: A 2D Riemann problem [29] computed by CO-DG-hr1e-3. $\Delta x=\Delta y=1 / 400$, Left: density. Right: pressure.

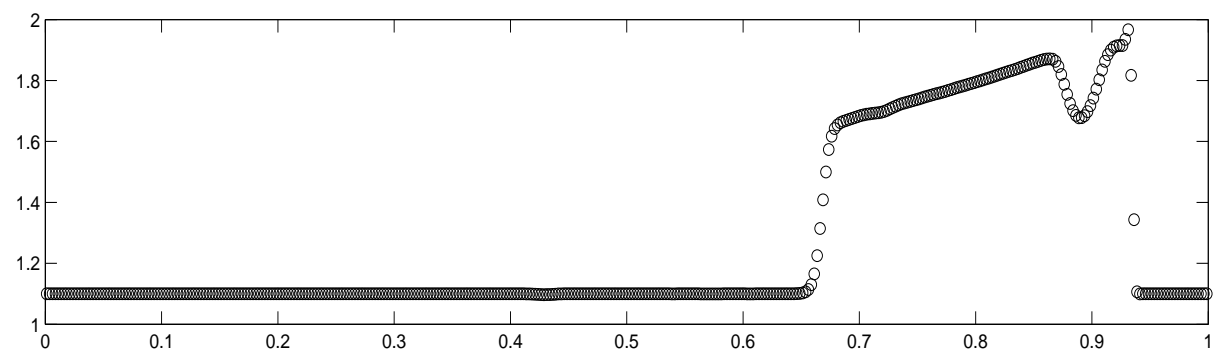

Figure 9: A 2D Riemann problem [29]. Density profile along $y=1 / 3$.

a CFL factor $0.4, \Delta t^{n}=0.99 \Delta \tau^{n}$. We can see in the lower graph (the cross-section density profile along $y=1 / 3$ ) that the computed result is non-oscillatory.

\section{Concluding Remarks and a Plan for Future Work}

In this paper we have developed a central discontinuous Galerkin method based on staggered overlapping cells, with a numerical viscosity which stays bounded when the time step size goes to zero. Time discretization is via the standard TVD Runge-Kutta method. We have also developed a multilayer hierarchical reconstruction procedure and used it as a limiter for our central DG scheme. The limiter is able to maintain the original order of accuracy and can effectively control spurious oscillations for discontinuous solutions. In future work we will generalize the method to convection diffusion equations, improve the limiter by applying troubled-cell indicators, and also study further the hierarchical reconstruction procedure as a limiter for the regular DG methods and finite volume schemes. A stability analysis and error estimates for the central DG scheme as well as a comparison between the regular DG and central DG schemes will also be performed.

The examples reported in the paper are aimed to show the flexibility of the new approach to use with a Runge-Kutta method, and its capability to handle small time steps, without introducing excessive numerical dissipation. The more efficient way to overcome the small time step restriction 

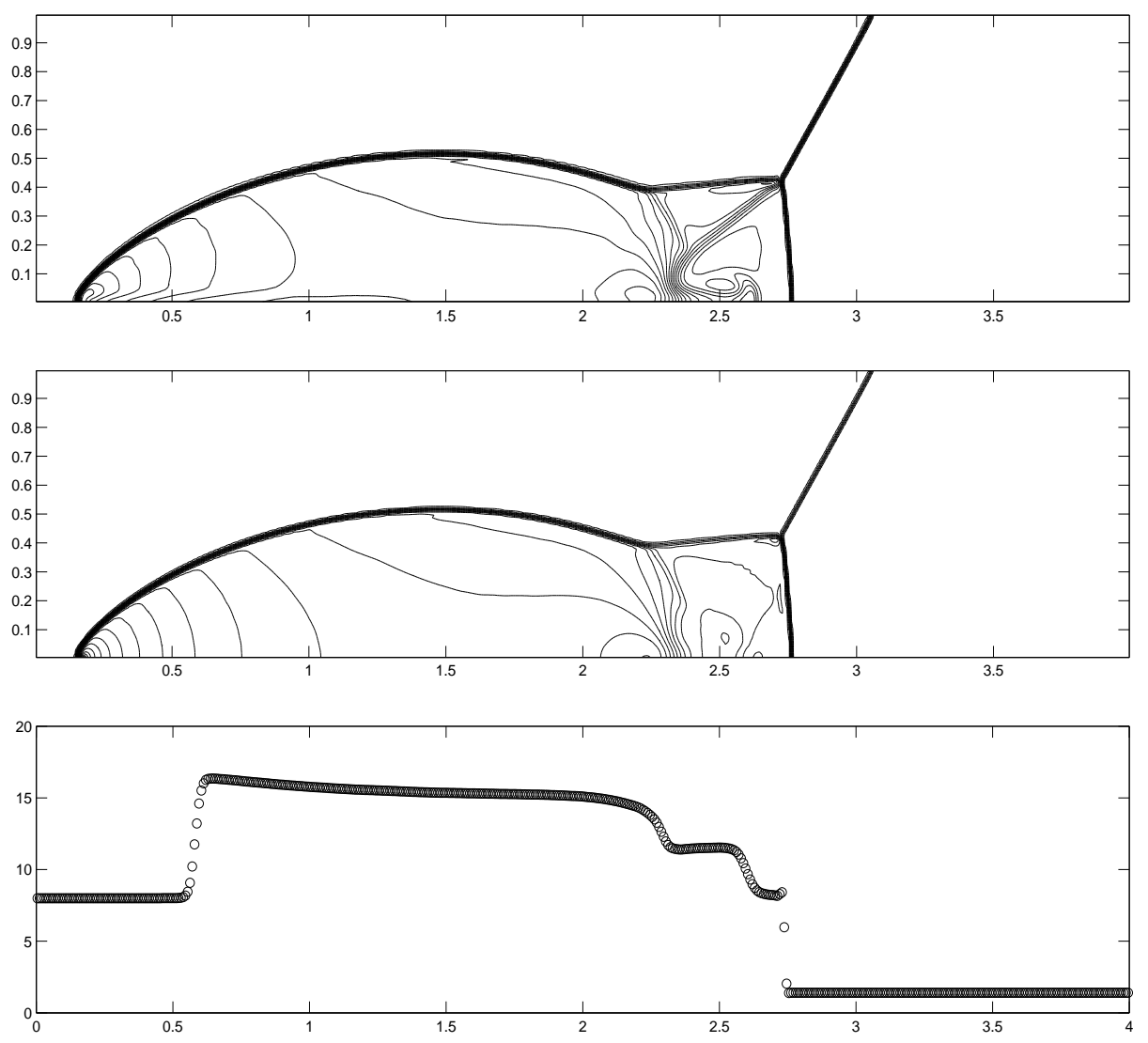

Figure 10: Double Mach reflection computed by CO-DG-hr1e-3. $\Delta x=\Delta y=1 / 120$. Top: density contours; Middle: pressure contours; Bottom: density cut along the line $y=1 / 3$.

with the presence of a diffusion term is to use the implicit-explicit time discretization, e.g. Ascher et. al. [4], Kennedy and Carpenter [24] and Liotta, Romano and Russo [33], which treats the advection part explicitly, and the diffusion part implicitly, thus avoiding the $O\left(\Delta x^{2}\right)$ stability restriction on the time step due to the diffusion term; or to use a fast explicit Runge-Kutta solver, e.g. Lebedev [30], Medovikov [39].

Even though in all the numerical examples, the reconstruction is performed componentwisely, we have also performed some preliminary tests on the non-oscillatory hierarchical reconstruction with local characteristic decomposition and have not found any significant difference. We plan to conduct more careful study on this subject in the future.

\section{REFERENCES}

[1] R. Abgrall, On essentially non-oscillatory schemes on unstructured meshes: analysis and implementation, J. Comput. Phys., 114 (1994), pp. 45-58.

[2] P. Arminjon And A. ST-CYR, Nessyahu-Tadmor-type central finite volume methods without predictor for $3 d$ cartesian and unstructured tetrahedral grids, Appl. Numer. Math., 46 (2003), pp. 135-155.

[3] P. Arminjon, M. C. Viallon, A. Madrane, and L. Kaddouri, Discontinuous finite elements and 2-dimensional finite volume versions of the Lax-Friedrichs and Nessyahu-Tadmor difference schemes for compressible flows on unstructured grids, CFD Review, (M. Hafez and K. Oshima, Eds), John Wiley, (1997), pp. 241-261.

[4] U. Ascher, S. Ruuth, And R. J. Spiteri, Implicit-explicit Runge-Kutta methods for time dependent partial differential equations, Appl. Numer. Math., 25 (1997), pp. 151-167. 
[5] J. BAlBAS AND E. TADMOR, CentPack: A package for high-resolution central schemes for nonlinear conservation laws and related problems, http://www.cscamm.umd.edu/centpack.

[6] F. Bianco, G. Puppo, And G. Russo, High-order central schemes for hyperbolic systems of conservation laws, SIAM J. Sci. Comput., 21 (1999), pp. 294-322.

[7] R. Biswas, K. Devine, And J. Flaherty, Parallel, adaptive finite element methods for conservation laws, Appl. Numer. Math., 14 (1994), pp. 255-283.

[8] J. Boris AND D. Book, Flux corrected transport I, SHASTA, a fluid transport algorithm that works, J. Comput. Phys., 11 (1973), pp. 38-69.

[9] N. Chevaugeon, J. Xin, P. Hu, X. Li, D. Cler, J. Flaherty, And M. Shephard, Discontinuous Galerkin methods applied to shock and blast problems, J. Sci. Comput., 22/23 (2005), pp. 227-243.

[10] B. Cockburn, An introduction to the discontinuous Galerkin method for convection-dominated problems, advanced numerical approximation of nonlinear hyperbolic equations (Cetraro, 1997), Lecture Notes in Math., Springer, Berlin., 1697 (1998), pp. 151-268.

[11] B. Cockburn, S.-Y. Lin, And C.-W. Shu, TVB Runge-Kutta local projection discontinuous Galerkin finite element method for conservation laws III: one dimensional systems, J. Comput. Phys., 84 (1989), pp. 90-113.

[12] B. Cockburn, S.-C.Hou, And C.-W. Shu, The Runge-Kutta local projection discontinuous Galerkin finite element method for conservation laws, Math. Comp., 54 (1990), pp. 545-581.

[13] B. Cockburn And C.-W. Shu, TVB Runge-Kutta local projection discontinuous Galerkin finite element method for conservation laws II, general framework, Math. Comp., 52 (1989), pp. 411-435.

[14] - The Runge-Kutta local projection $p^{1}$-discontinuous-Galerkin finite element method for scalar conservation laws, RAIRO Model. Math. Anal. Numer., 25 (1991), pp. 337-361.

[15] — The Runge-Kutta discontinuous Galerkin method for conservation laws V: multidimensional systems, J. Comput. Phys., 141 (1998), pp. 199-224.

[16] P. Colella ANd P. Woodward, The piecewise parabolic method (PPM) for gas-dynamical simulation, J. Comput. Phys., 54 (1984), pp. 174-201.

[17] J. Glimm, X.-L. Li, Y. LiU, Z. Xu, And N. Zhao, Conservative front tracking with improved accuracy, SIAM J. Numer. Anal., 41 (2003), pp. 1926-1947.

[18] D. Gottlieb, C.-W. Shu, And E. Tadmor, Strong stability-preserving high order time discretization methods, SIAM Review, 43 (2001), pp. 89-112.

[19] A. HaRten, High resolution schemes for hyperbolic conservation laws, J. Comput. Phys., 49 (1983), pp. $357-393$.

[20] A. Harten, B. Engquist, S. Osher, and S. R. Chakravarthy, Uniformly high order accuracy essentially non-oscillatory schemes III, J. Comput. Phys., 71 (1987), pp. 231-303.

[21] G.-S. JiAng, D. Levy, C.-T. Lin, S. Osher, AND E. TADMOR, High-resolution non-oscillatory central schemes with non-staggered grids for hyperbolic conservation laws, SIAM J. Numer. Anal., 35 (1998), p. 2147.

[22] G.-S. Jiang And E. TADmor, Non-oscillatory central schemes for multidimensional hyperbolic conservation laws, SIAM J. Sci. Comput., 19 (1998), pp. 1892-1917.

[23] S. Jin AND Z. XIN, The relaxation schemes for systems of conservation laws in arbitrary space dimensions, Comm. Pure Appl. Math., 48 (1995), pp. 235-276.

[24] C. A. Kennedy And M. H. CarPenter, Additive Runge-Kutta schemes for convection-diffusion-reaction equations, Appl. Numer. Math., 44 (2003), pp. 139-181.

[25] A. Kurganov And D. Levy, A third-order semi-discrete central scheme for conservation laws and convectiondiffusion equations, SIAM J. Sci. Comput., 22 (2000), pp. 1461-1488.

[26] A. Kurganov and C.-T. Lin, On the reduction of numerical dissipation in central-upwind schemes, Commun. Comput. Phys., 2 (2007), pp. 141-163.

[27] A. Kurganov, S. Noelle, And G. Petrova, Semidiscrete central-upwind schemes for hyperbolic conservation laws and Hamilton-Jacobi equations, SIAM J. Sci. Comput., 23 (2001), pp. 707-740.

[28] A. Kurganov And E. TAdmor, New high-resolution central schemes for nonlinear conservation laws and convection-diffusion equations, J. Comput. Phys., 160 (2000), pp. 241-282.

[29] P. LAX AND X.-D. LIU, Solution of two dimensional riemann problem of gas dynamics by positive schemes, SIAM J. Sci. Comput., 19 (1998), pp. 319-340.

[30] V. I. LEBedev, Explicit difference schemes for solving stiff systems of odes and pdes with complex spectrum, Russian J. Numer. Anal. Math. Modelling, 13 (1998), pp. 107-116.

[31] P. Lesaint And P. A. RAviart, On a finite element method for solving the neutron transport equation, Mathematical Aspects of Finite Elements in Partial Differential Equations, C. de Boor. ed., Academic Press, NY, (1974).

[32] D. Levy, G. Puppo, And G. Russo, A fourth-order central WENO scheme for multidimensional hyperbolic systems of conservation laws, SIAM J. Sci. Comput., 24 (2002), pp. 480-506.

[33] S. F. Liotta, V. Romano, And G. Russo, Central schemes for balance laws of relaxation type, SIAM J. Numer. anal., 38 (2000), pp. 1337-1356. 
[34] X. D. LiU AND S. OsheR, Convex ENO high order multi-dimensional schemes without field by field decomposition or staggered grids, J. Comput. Phys., 142 (1998), pp. 304-330.

[35] X. D. Liu, S. Osher, And T. Chan, Weighted essentially non-oscillatory schemes, J. Comput. Phys., 115 (1994), pp. $408-463$.

[36] X.-D. LIU AND E. TADMOR, Third order nonoscillatory central scheme for hyperbolic conservation laws, Numer. Math., 79 (1998), pp. 397-425.

[37] Y. Liu, Central schemes on overlapping cells, J. Comput. Phys., 209 (2005), pp. 82-104.

[38] — Central schemes and central discontinuous Galerkin methods on overlapping cells, Conference on Analysis, Modeling and Computation of PDE and Multiphase Flow, Stony Brook, NY., (Aug., 2004).

[39] A. A. Medovikov, High order explicit methods for parabolic equations, BIT, 38 (1998), pp. 372-390.

[40] H. Nessyahu and E. Tadmor, Non-oscillatory central differencing for hyperbolic conservation laws, J. Comput. Phys., 87 (1990), pp. 408-463.

[41] J. QIU AND C.-W. SHU, Hermite WENO schemes and their application as limiters for Runge-Kutta discontinuous galerkin method. II. two dimensional case., Comput. \& Fluids, 34 (2005), pp. 642-663.

[42] — - Runge-Kutta discontinuous Galerkin method using WENO limiters, SIAM J. Sci. Comput., 26 (2005), pp. 907929.

[43] R. SANDERS AND A. WEISER, High resolution staggered mesh approach for nonlinear hyperbolic systems of conservation laws, J. Comput. Phys., 101 (1992), pp. 314-329.

[44] C.-W. SHu, Essentially non-oscillatory and weighted essentially non-oscillatory schemes for hyperbolic conservation laws, Advanced Numerical Approximation of Nonlinear Hyperbolic Equations, A. Quarteroni, ed., Lecture Notes in Math.,Springer, Berlin., 1697 (1998).

[45] C.-W. Shu AND S. Osher, Efficient implementation of essentially non-oscillatory shock-capturing schemes, J. Comput. Phys., 77 (1988), pp. 439-471.

[46] — Efficient implementation of essentially nonoscillatory shock-capturing schemes, II, J. Comput. Phys., 83 (1989), pp. 32-78.

[47] H.-Z. TANG AND T. TANG, Adaptive mesh methods for one- and two-dimensional hyperbolic conservation laws, SIAM J. Numer. Anal., 41 (2003), pp. 487-515.

[48] B. VAN LEeR, Towards the ultimate conservative difference scheme I, Lecture Notes in Phys., 18 (1973), pp. $163-168$.

[49] — Towards the ultimate conservative difference scheme II. monotonicity and conservation combined in a second order scheme, J. Comput. Phys., 14 (1974), pp. 361-370.

[50] P. Woodward And P. Colella, The numerical simulation of two-dimensional fluid flow with strong shocks, J. Comput. Phys., 54 (1984), pp. 115-173. 
(Yingjie Liu)

School of Mathematics

Georgia Institute of Technology

Atlanta, GA 30332-0160

E-mail address: yingjie@math.gatech.edu

URL: http://www . math.gatech.edu/ ${ }^{\text {yingjie/ }}$

(Chi-Wang Shu)

Division of Applied Mathematics

BROWN UNIVERSITY

Providence RI 02912

E-mail address: shu@dam.brown.edu

URL: http://www.dam. brown.edu/people/shu/

(Eitan Tadmor)

Department of Mathematics, Institute for Physical Science and Technology

and Center of Scientific Computation And Mathematical Modeling (CSCAMM)

UNIVERSiTy OF MARYLAND

College PARK, MD 20742 USA

E-mail address: tadmor@cscamm.umd.edu

$U R L:$ http://www.cscamm.umd.edu/people/faculty/tadmor

(Mengping Zhang)

DePARTMENT OF MATHEMATICS

University of Science and Technology of China

Hefei, Anhui 230026, China

E-mail address: mpzhang@ustc.edu.cn 